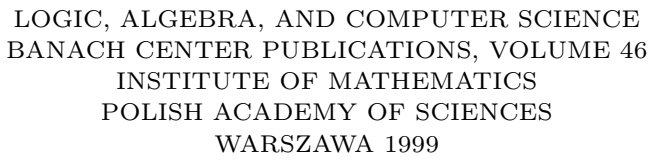

\title{
A THEORY OF REFINEMENT STRUCTURE OF HEDGE ALGEBRAS AND ITS APPLICATIONS TO FUZZY LOGIC
}

\author{
NGUYEN CAT HO \\ Institute of Information Technology, NCST \\ Hanoi, Vietnam \\ E-mail: ncho@ioit.ncst.ac.vn \\ HUYNH VAN NAM \\ Department of Mathematics, Quinhon University of Pedagogy \\ 170 Nguyen Hue, Quinhon, Vietnam
}

\begin{abstract}
In [13], an algebraic approach to the natural structure of domains of linguistic variables was introduced. In this approach, every linguistic domain can be interpreted as an algebraic structure called a hedge algebra. In this paper, a refinement structure of hedge algebras based on free distributive lattices generated by linguistic hedge operations will be examined in order to model structure of linguistic domains more properly. In solving this question, we restrict our consideration to the specific hedge algebras called PN-homogeneous hedge algebras. It is shown that any PN-homogeneous hedge algebra can be refined to a refined hedge algebra (RHA, for short) and every RHA with a chain of the primary generators is a distributive lattice. Especially, we shall examine RHAs with exactly two distinct generators, which will be called symmetrical RHAs. Furthermore, in the symmetrical RHAs of the linguistic truth variable, we are able to define negation and implication operation, which, according to their properties, may be interpreted as logical negation and implication in a kind of fuzzy logic called linguisticvalued logic. Some elementary properties of these operations will be also examined. This yields a possibility to construct a method in linguistic reasoning, which is based on linguistic-valued fuzzy logic corresponding to the symmetrical RHAs of the linguistic truth variable.
\end{abstract}

1. Introduction. It is known that humans reason by means of their own language and they can choose and decide alternatives by evaluating semantics of linguistic terms. The fundamental elements in human reasoning are sentences normally containing vague

1991 Mathematics Subject Classification: 03B52, 03G10, 03G25, 68T27.

The research was supported in part by The Vietnam National Program for Basic Research in Natural Sciences.

The paper is in final form and no version of it will be published elsewhere. 
concepts, and these sentences have implicitly or explicitly a truth degree, which is often expressed also by linguistic terms such as true, very true, more or less true, approximately true, false, very false, etc. In connection with this, Rinks wrote in [22] that "verbal coding is a human way of repackaging material into a few chunks of rich information. Natural language is rather unique in this characteristic. Until recently, a unified theory for manipulating in a strict mathematical sense non-numerical-valued variables, such as linguistic terms, did not exist."

Furthermore, it is well-known that Boolean algebras, Post algebras, 3-valued and multiple-valued Łukasiewicz algebras, etc. are algebraic foundations of classical or nonclassical logics (see, e.g., $[4,8,20,21,23])$. In this direction, we want to look for an algebraic structure for fuzzy logic based on a suitable structure of truth vague concepts. It is known that L.A. Zadeh introduced and examined fuzzy logic based on the notion of linguistic variables. A linguistic variable is characterised by a quintuple $(X, T(X), U, G, M)$, where $X$ is the name of the variable; $T(X)$ denotes the term-set of $X, U$ is a universe of discourse of the base variable, $G$ is a syntactic rule for generating linguistic terms of $T(X)$, and $M$ is a semantic rule which is a mapping assigning to each linguistic term a fuzzy set on $U$. Recall that a fuzzy set of $U$ is an element of the set $F(U,[0,1])$ of all functions from $U$ to the unit interval $[0,1]$. In our approach, each term is associated with an element in an RHA, and its meaning is expressed through the structure of such an RHA.

In the papers initiated by Ho \& Wechler [5,13], an algebraic approach to the natural structure of domains of linguistic variables was examined. As mentioned above, the main aim of our investigation is to find an appropriate algebraic structure for fuzzy logic and fuzzy reasoning, which could model human reasoning in an advantageous way. There are three main reasons for pursuing the research in this direction. The first one is that the domains of linguistic variables can be embedded into mathematical structure: the lattice structure, which is well-known in applications to logic. In such a structure, these domains can be ordered in a reasonable way, based on intuitive meanings of vague concepts. The second one is that there exists a natural demand to find a mathematical method for manipulating immediately linguistic terms as depicted above. The third one is that the way ones interpret the meaning of linguistic terms as fuzzy sets loses the natural ordering structure of linguistic domains.

In [13], an axiomatization for the so-called hedge algebras was introduced. Recall that the axiomatization is based on a detailed discussion about the general characteristics of linguistic hedges and vague concepts in natural language. The idea of this research was suggested from the research works of Zadeh and Lakoff [18,26,28], in which linguistic hedges and vague concepts are considered within the framework of fuzzy set theory.

In the algebraic approach, every linguistic domain can be interpreted as an algebra $A X=(X, G, H, \leq)$, where $(X, \leq)$ is a poset and $G$ is a set of the primary generators and $H$ is a set of unary operations representing linguistic hedges under consideration. In [14], hedge algebras were extended by introducing two additional operations corresponding to infimum and supremum of the so-called concept category of an element $x$, i.e. the set $H(x)$, which is generated from $x$ by means of hedge operations.

It is shown that every extended hedge algebra (EHA, for short) with a lattice of the primary generators is a lattice and they can be used as an algebraic basis for a fuzzy logic 
called linguistic-valued logic (see, e.g., Ho [5-7]). However, many linguistic terms, which contain logical connectives disjunction "or" and/or conjunction "and" like 'Approximately True or Possibly True', cannot be reasonably expressed by elements of hedge algebras. The reason lies in the fact that although we can define in these algebras operations of join $\cup$ and meet $\cap$, which may be interpreted as disjunction "or" and conjunction "and", but, in our opinion, these structures are rather rough. For example, let us consider the set of all possible truth values

$T=\{$ true, false, very true, very false, approximately true, possibly true, approximately true or possibly true, approximately true and possibly true,...\}

We can see that the above linguistic value "approximately true or possibly true" will be expressed by "true" in the structure of EHA of the set of linguistic truth values, i.e. they define the same element in this algebra, which is clearly unsuitable in nature. Another disadvantage is that EHA, in general, are not distributive and hence we are not able to discuss the disjunction and conjunction normal forms.

In this paper we shall introduce some new axioms and obtain a class of algebras called refined hedge algebras (RHAs, for short), which have a finer structure than that of hedge algebras.

The paper is organised as follows: In Section 2 we shall present a way of constructing the distributive lattices of hedge operations. We shall introduce in Section 3 an axiomatization for RHA. A characterization to determine the relative position of elements in an RHA and some fundamental properties of this structure will be examined. The main property, which says that every RHA with a chain of the primary generators is a distributive lattice, will be studied in Section 4. In Section 5, RHAs with exactly two distinct generators called symmetrical RHAs will be examined. As a consequence, these RHAs are distributive lattices. Moreover, in Section 6 we shall point out that, in the finite symmetrical RHAs of the domains of the linguistic truth variable, we are able to define negation operation and implication operation, which may be interpreted as logical negation and implication. Some elementary properties of these operations will be also presented. Finally, some concluding remarks will be given in Section 7 .

2. Distributive lattices of hedge operations. As mentioned in the previous section, the main aim of our investigation is to find a finer structure than that of hedge algebras. In order to construct this structure, we need some preparations.

First we shall recall some notions and notations introduced in [1]. Let $P$ be a partial ordered set (poset, for short).

Definition 2.1. An element $a$ is said to cover an element $b$ in a poset $P$, if $a>b$ and there is no $x \in P$ such that $a>x>b$.

By the order $o(P)$ of a poset $P$ we mean the number of its elements, and if this number is finite, $P$ is called a finite poset. Denote by $l(P)$ the length of a poset $P$.

In a poset $P$ of finite length with the least element denoted by $O$, the height of an element $x \in P$ is, by definition, the least upper bound of the length of the chains $O=$ $x_{0}<x_{1}<\ldots<x_{n}=x$ between $O$ and $x$, and it is denoted by $h(x)$. If $P$ has the greatest element, denoted by 1 , then clearly $h(1)=l(P)$. Clearly also $h(x)=1$ iff $x$ covers $O$. 
Definition 2.2. A poset $P$ is said to be graded if there exists a function from $P$ to the set $Z$ of all integers with the natural ordering, $g: P \rightarrow Z$, such that:

G1. $x>y$ implies $g(x)>g(y)$.

G2. If $x$ covers $y$ then $g(x)=g(y)+1$.

Such a function $g$ is called the graded function of $P$. It is known that any modular lattice of finite length is graded by its height function $h(x)$.

Let $L$ be a modular lattice of finite length, we can define a relation $R$ on $L$ as follows:

$$
\forall x, y \in L,(x, y) \in R \text { iff } h(x)=h(y)
$$

It is easily shown that $R$ is an equivalence relation and then we have $L=\bigcup_{i=0}^{l(L)} L_{i}$ where $L_{i}=\{x \in L / h(x)=i\}, i=0, \ldots, l(L)$, are the equivalence classes of the relation $R$. Clearly, $L_{0}=\{0\}$ and $L_{l(L)}=\{1\}$.

In order to model the structure of sets of linguistic hedges, we need the following assumption, which describes the fact that any two hedges belonging to two different equivalence classes are always comparable:

(C0) Either $x>y$ or $x<y$ for any $x \in L_{i}$ and $y \in L_{j}$ and $i \neq j$.

To illustrate this, the reader can see the classes $L_{1}=\{I\}, L_{2}=\{A, P, M L\}$ and $L_{3}=\{L\}$ as in Figure 3 .

It is not difficult to see that the following holds:

Proposition 2.1. Let $L$ be a modular lattice of finite length satisfying $(\mathrm{C} 0)$. Then the following condition holds:

If $o\left(L_{i}\right)>1$ for an index $i \in\{1, \ldots, l(L)-1\}$ then $o\left(L_{i-1}\right)=o\left(L_{i+1}\right)=1$. Moreover, if we denote $e\left(L_{i+1}\right)$ and $e\left(L_{i-1}\right)$ the single element of $L_{i+1}$ and $L_{i-1}$, respectively, then $e\left(L_{i+1}\right)=\vee_{x \in L_{i}} x$ and $e\left(L_{i-1}\right)=\wedge_{x \in L_{i}} x$, where $\vee$ and $\wedge$ are supremum and infimum in $L$, respectively.

We proceed now to consider a hedge algebra ${ }^{1} A X=(X, G, H, \leq)$, where $(X, \leq)$ is a poset, $G$ is a set of the primary generators and $H$ is a set of unary operations representing linguistic hedges under consideration. It is assumed that $H$ can be decomposed into two disjoint subsets $H^{+}$and $H^{-}$such that $H^{+}+I$ and $H^{-}+I$ are finite modular lattices, where $I$ is the identity, i.e. $I x=x$ for every $x$ in $X$, and considered as their zero-element. An example for this can be seen in Figure 3.

We will denote by $N^{+}$and $N^{-}$the lengths of $H^{+}+I$ and $H^{-}+I$, respectively. Suppose that $g^{+}$and $g^{-}$are the graded functions of $H^{+}+I$ and $H^{-}+I$, respectively.

Unless stated otherwise, in the sequel we shall always adopt the assumption that $H^{+}+I$ and $H^{-}+I$ are finite modular lattices and satisfy the condition (C0). From now on, $V$ and $L$ stand for the unit-operations in $H^{+}+I$ and $H^{-}+I$, respectively. Hence, we have $g^{+}(V)=N^{+}, g^{-}(L)=N^{-}$and

$$
\begin{aligned}
& H^{+}+I=\bigcup_{i=0}^{N^{+}} H_{i}^{+} \text {where } H_{i}^{+}=\left\{h \in H^{+}+I / g^{+}(h)=i\right\}, \\
& H^{-}+I=\bigcup_{i=0}^{N^{-}} H_{i}^{-} \text {where } H_{i}^{-}=\left\{h \in H^{-}+I / g^{-}(h)=i\right\} .
\end{aligned}
$$

\footnotetext{
${ }^{1}$ See Ho \& Wechler [14] for more details.
} 
We shall now construct lattices, which are "freely" generated from $\mathrm{H}^{+}+I$ and $\mathrm{H}^{-}+I$.

Let us consider $H^{+}+I$. Assume that for some index $i \in 1, \ldots, N^{+}, o\left(H_{i}^{+}\right)>1$, and $H_{i}^{+}=\left\{h_{1}^{i}, \ldots, h_{n}^{i}\right\}$. By Proposition 2.1, the sets $H_{i+1}^{+}=\left\{h^{i+1}\right\}$ and $H_{i-1}^{+}=\left\{h^{i-1}\right\}$ are single-element sets. For such $i$, the ordering relationships between the elements of $H_{i-1}^{+}, H_{i}^{+}, H_{i+1}^{+}$, can be expressed as in Figure 1 . Note that, as assumed above, there exists a natural ordering relation between classes $H_{i}^{+}$and $H_{i}^{+} \leq H_{j}^{+}$iff $i \leq j$, where $H_{i}^{+} \leq H_{j}^{+}$means that $x \leq y$ for every $x \in H_{i}^{+}$and $y \in H_{j}^{+}$.

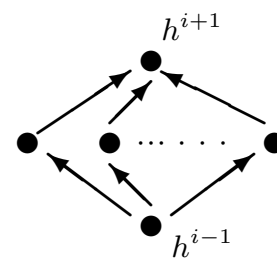

Fig. 1

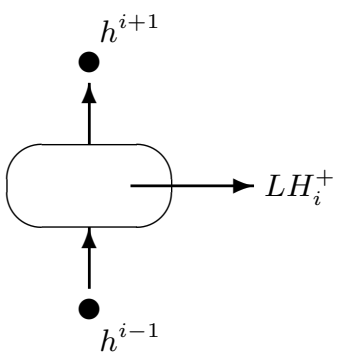

Fig. 2

Denote by $L_{i}^{+}=\left(L\left(H_{i}^{+}\right), \vee, \wedge\right)$ the free distributive lattice ${ }^{2}$ generated from the incomparable elements $h_{1}^{i}, \ldots, h_{n}^{i}$ of $H_{i}^{+}$. Particularly, for an index $i$ such that $o\left(H_{i}^{+}\right)=1$, we have $L H_{i}^{+}=H_{i}^{+}$. Put $L_{u}^{+}=\bigcup_{i=0}^{N^{+}} L H_{i}^{+}$and $L H_{i}^{+}+I=\left(L_{u}^{+}, H^{+}+I, \vee, \wedge\right) . L u^{+}$ becomes a distributive lattice under the ordering relation induced by the ordering relation of the lattices $\mathrm{LH}_{i}^{+}$and that defined between classes $H_{i}^{+}$, i.e. we have $\mathrm{LH}_{i}^{+} \leq \mathrm{LH}_{j}^{+}$, for any $i, j$ such that $i \leq j$. Figure 2 shows a picture of a segment of the constructed lattice $L H^{+}+I$, where $o\left(H_{i}^{+}\right)>1$.

In an analogous way, we can construct the lattice $L H^{-}+I=\left(L_{u}^{-}, H^{-}+I, \vee, \wedge\right)$, generated from $H^{-}+I$. Here, there is no confusion, because $H^{+}$and $H^{-}$are assumed to be disjoint and hence, so are $L H^{+}$and $L H^{-}$, where $L H^{+}=L H^{+}+I \backslash\{I\}$ and $L H^{-}=L H^{-}+I \backslash\{I\}$. Thus, we have the following

TheOrem 2.1. $L H^{+}+I=\left(L_{u}^{+}, \vee, \wedge, I, V, \leq\right)$ and $L H^{-}+I=\left(L_{u}^{-}, \vee, \wedge, I, L, \leq\right)$ are finite distributive lattices.

EXAMPLE 2.1. Let us consider the algebraic structure $A X=(X, G, H, \leq)$, in which $G=\{$ True, False $\}$ and $H^{+}=\{V, M\}$ and $H^{-}=\{L, A, P, M L\}$. Here, for short, $V$, $M, L, A, P, M L$ stand for Very, More, Little, Approximately, Possibly, More or Less, correspondingly, and $H^{+}+I$ and $H^{-}+I$ are lattices depicted in Figure 3. Clearly, $H^{+}+I$ and $H^{-}+I$ are finite modular lattices and satisfy condition (C0). By a construction as above, the distributive lattices $\mathrm{LH}^{+}+I$ and $\mathrm{LH}^{-}+I$ generated from $\mathrm{H}^{+}+I$ and $\mathrm{H}^{-}+I$, respectively, can be represented as in Figure 4, where

$x_{1}=P \vee M L, x_{2}=M L \vee A, x_{3}=A \vee P, u_{1}=x_{2} \wedge x_{3}, u_{2}=x_{3} \wedge x_{1}, u_{3}=x_{1} \wedge x_{2}$,
$y_{1}=P \wedge M L, y_{2}=M L \wedge A, y_{3}=A \wedge P, v_{1}=y_{2} \vee y_{3}, v_{2}=y_{3} \vee y_{1}, v_{3}=y_{1} \vee y_{2}$,
$E=(A \vee P) \wedge(P \vee M L) \wedge(M L \vee A)=(A \wedge P) \vee(P \wedge M L) \vee(M L \wedge A)$.

${ }^{2}$ See, e.g., Birkhoff [1]. 


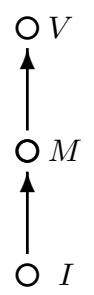

a) $H^{+}$

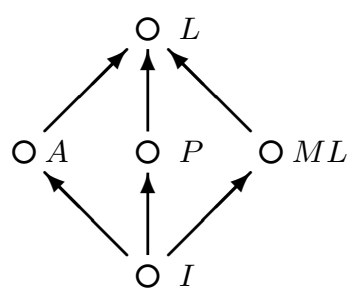

b) $H^{-}$

Fig. 3

a) $L H^{+}+I=H^{+}+I$

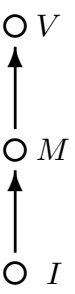

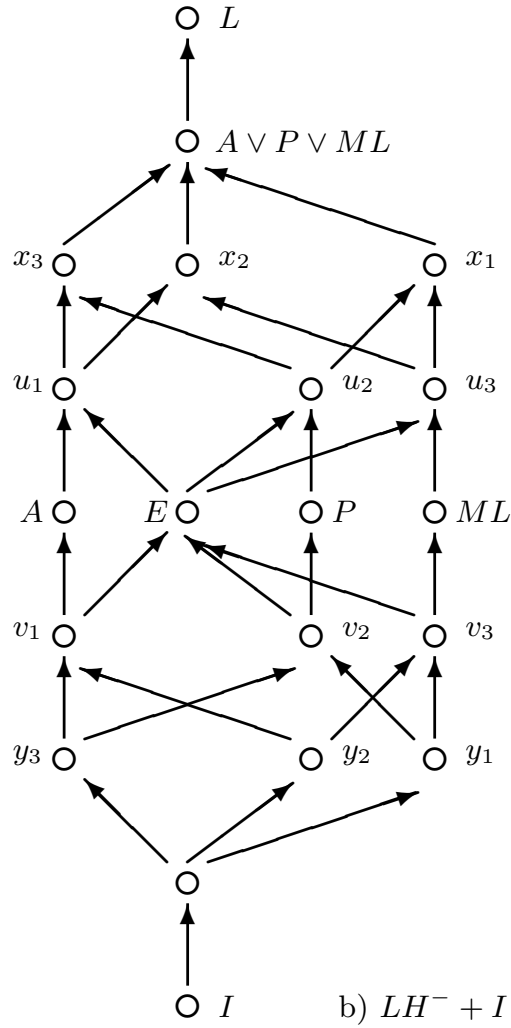

Fig. 4

3. An axiomatization for RHA and its elementary properties. Let us consider a hedge algebra $A X=(X, G, H, \leq)$, where $H^{+}+I$ and $H^{-}+I$ are finite modular lattices satisfying condition (C0). Suppose that $\mathrm{LH}^{+}+I$ and $\mathrm{LH}^{-}+I$ are distributive lattices, which are generated from $\mathrm{H}^{+}+I$ and $\mathrm{H}^{-}+I$, respectively, as presented in the previous section. Let $I^{+}=\left\{0,1, \ldots, N^{+}\right\}, I^{-}=\left\{0,1, \ldots, N^{-}\right\}$, and $S I^{+}=\left\{i \in I^{+} / o\left(H_{i}^{+}\right)>1\right\}$ and $S I^{-}=\left\{i \in I^{-} / o\left(H_{i}^{-}\right)>1\right\}$. For simplifying the formulation of some statements, in the sequel by ${ }^{c}$ ' we mean either '+' ${ }^{\prime}$ or ${ }^{\prime-}$, , and then for a statement containing, for instance, the notation $L H_{i}^{c}$ for some $i \in S I^{c}$, we mean the statement presents two instances obtained by substituting "c" in turn by "+" and "-". For example, under such 
convention we can state the following: for any $i \in S I^{c}, L H_{i}^{c}$ is the free distributive lattice generated from the incomparable elements of $H_{i}^{c}$ and is a sublattice of $L H^{c}+I$; and for $i \in I^{c} \backslash S I^{c}, L H_{i}^{c}$ is a single-element set, $L H_{i}^{c}=H_{i}^{c}$ and we have

$$
L H^{c}+I=\bigcup_{i=0}^{N^{c}} L H_{i}^{c} .
$$

Put $L H=L H^{+} \cup L H^{-} \cup\{I\}$.

Let us denote by $U O S$ the set of two elements $V$ and $L$, which are unit-operations in $L H^{+}+I$ and in $L H^{-}+I$, respectively. Denote by Nat the set of all non-negative integers. We introduce the following notion which will be used as an assumption throughout the paper:

Definition 3.1. A hedge algebra $A X=(X, G, H, \leq)$ is said to be $P N$-homogeneous, where PN is an abbreviation of Positive and Negative, provided that for any set $H_{i}^{c}$ if the unit operation $V$ in $H^{+}+I$ is positive ${ }^{3}$ (negative, resp.) w.r.t. a certain operation $h$ in $H_{i}^{c}$, then $V$ is also positive (negative resp.) w.r.t. any other one in $H_{i}^{c}$.

For example, the hedge algebra $A X=(X, G, H, \leq)$ in Example 2.1 is a PN-homogeneous hedge algebra. Since every hedge $h$ is a mapping from $X$ into $X$, the image of an element $x$ in $X$ under $h$ will be denoted by $h x$ instead of $h(x)$, for convenience. Thus, we can write $k h x$ instead of $k(h(x))$ for any $h, k \in L H$ and $x \in X$. According to our convention, for simplicity in formulating several statements as mentioned in [13], for any $h \in L H$, we define $h I x=I x=x$, i.e. when $I$ occurs in an expression explicitly, any $h$ applying to $I x$ will have no effect.

The following definition gives us the semantics of the inequality $h>k$, which describes a property in the natural language saying that a hedge is stronger than another one, e.g. Little is stronger than Possibly.

Definition 3.2. An algebra $A X=(X, G, L H, \leq)$ is said to be semantically consistent if for any $h, k \in L H^{c}+I, x \in X$ and $h x \neq k x, h$ and $k$ are comparable in $L H^{c}+I$ iff $h x$ and $k x$ are comparable and if $h>k$ then $h x>k x$, when $h x>x$, and $h x<k x$, when $h x<x$.

Throughout the paper we always assume the considered algebra $A X$ satisfies the semantic consistency in Definition 3.2. For any two hedges $h, k$ in $L H$, if the statement $x \leq h x$ iff $k x \leq x$ holds, for every $x$ in $X$, then $h$ and $k$ are said to be converse, or $h$ is converse to $k$ and vice-versa. If the statement $x \leq h x$ iff $x \leq k x$ holds, for every $x$ in $X$, then $h$ and $k$ are said to be compatible.

Consider an algebra $A X=(X, G, L H, \leq)$, where $G$ is a set of zero-argument operations, $L H$ is a set of one-argument operations.

For every $x \in X, L H(x)$ denotes the set of all elements generated from $x$ by means of operations in $L H$. More generally, for $Y \subset X$ and $H^{\prime} \subset L H, H^{\prime}(Y)$ denotes the subset of $X$ generated from the elements in $Y$ by means of the operations in $H^{\prime}$. Particularly, $H^{\prime}(I x)=\{x\}$. As usual, $L H^{*}$ denotes the set of all strings of hedges in $L H$.

\footnotetext{
${ }^{3}$ See Ho \& Wechler [13].
} 
REMARK 3.1. From the construction of the lattices $L H^{+}+I$ and $L H^{-}+I$, it can be seen that the lattices $L H^{+}+I$ and $L H^{-}+I$ also satisfy condition (C0), in which the notations $L_{i}$ and $L_{j}$ are replaced with $L H_{i}^{c}$ and $L H_{j}^{c}$, respectively.

Now, we introduce an axiomatization for a refinement structure of hedge algebras.

Definition 3.3. An algebra $A X=(X, G, L H, \leq)$ is said to be a refined hedge algebra (or, briefly, RHA), if $(H(G), G, H, \leq)$ is a PN-homogeneous hedge algebra and the following conditions hold:

(R1) Every operation in $\mathrm{LH}^{+}$is a converse operation of the operations in $\mathrm{LH}^{-}$. In addition, the unit operation $V$ in $\mathrm{LH}^{+}$is either positive or negative w.r.t. any operations in $L H$.

(R2) If $u$ and $v$ are independent, i.e. $u \notin L H(v)$ and $v \notin L H(u)$, then $x \notin L H(v)$ for any $x \in L H(u)$. For $x \neq h x, x \notin L H(h x)$. Especially, if $a, b \in G$ and $a<b$ then $L H(a)<L H(b)$.

(R3) If $h x$ and $k x$ are incomparable, then so are any elements $u \in L H(h x)$ and $v \in L H(k x)$. For any $h \neq k$ and $h x \leq k x$ :

(i) If $h, k \in L H_{i}^{c}$, for $i \in S I^{c}$, and $h x \neq k x$ then $\delta h x<\delta k x$, for any string of hedges $\delta$.

Furthermore, for any $y \in L H(k x)$ such that $y \nsupseteq \delta k x$, $\delta h x$ and $y$ are incomparable, and for any $z \in L H(h x)$ such that $z \not \leq \delta h x, \delta k x$ and $z$ are incomparable.

(ii) If both $h$ and $k$ are different from $I$ and do not belong to the same sublattice $L H_{i}^{c}$ or $h x=k x$, then $h^{\prime} h x \leq k^{\prime} k x$, for any $h^{\prime}, k^{\prime} \in U O S$.

(iii) If $h x \neq k x$ then $h x$ and $k x$ are independent.

(R4) If $u \in L H(x)$ and suppose that $u \notin L H(h x)$, for any $h \in L H_{i}^{c}, i \in I^{c}$ then $u \geq v$ $(u \leq v)$ for $v \in L H(h x)$ implies $u \geq h^{\prime} v\left(u \leq h^{\prime} v\right)$, for any $h^{\prime} \in U O S$.

Now, we give an intuitive illustration of some axioms in Definition 3.3. (R2) describes a linguistic property saying that, for instance, if $u=$ Possibly true and $v=$ Approximately true, then $u$ and $v$ are independent and any term $x$ generated from $u$, e.g. $x=$ Very Poss. true, must inherit the meaning of Possibly true and, hence, it cannot be generated from Approximately true. (R4) models the following semantic property of natural language: if $h x=$ Approximately True and $u$ satisfies the condition in (R4) with $v=$ Very Approximately true $\geq u$ then $u$ must be a term generated from Little true and hence $u \leq h^{\prime} v$, where $h^{\prime}$ is either Very or Little. The statement (i) of (R3) is the basis to establish a partially ordering between the elements presented in Figure 5, that suits our intuition. The statement (ii) of (R3) guarantees that elements of L(A,P,ML)(True) in Figure 6 must be less than I.True = True and greater than L.True. The statement (iii) of (R3) states that a linguistic meaning generated from $h x$ is not deduced from $k x$ and vice-versa.

Note that the first part of (ii) in (R3) can be reformulated to include the case where one of $h$ and $k$ is to be the identity $I$, based on our convention upon $I$. But, then, it will be a consequence of (R4).

EXAMPLE 3.1. Let us consider an algebraic structure $A X=(X, G, L H, \leq)$, where $H=\{V, M, L, A, P, M L\}$ is the set of hedges considered in Example 2.1. For every hedge 
P.True:= Possibly True A.True:= Approx. True $\mathrm{M}:=$ More; $\mathrm{V}:=$ Very

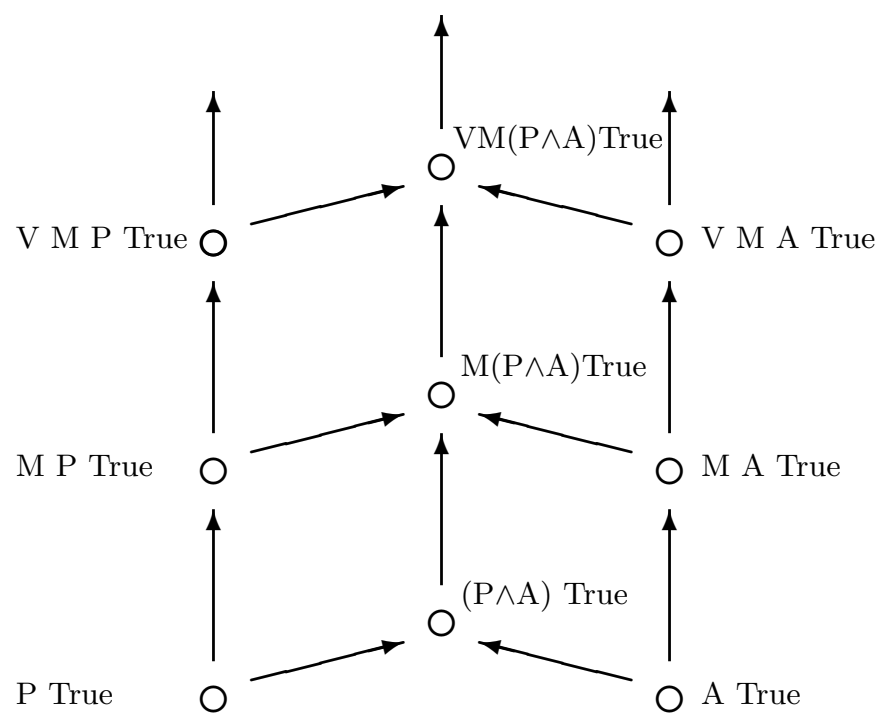

Fig. 5
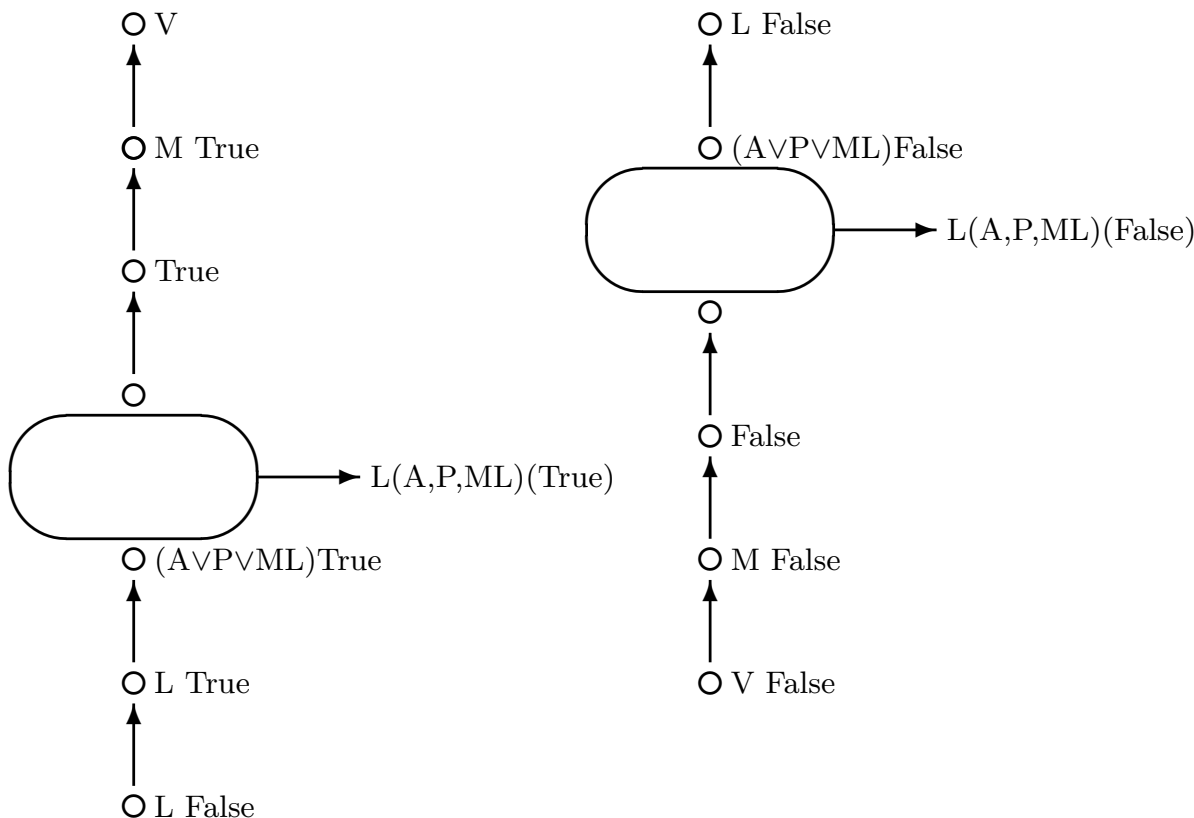

Fig. 6

operation $h$ in $L H, h$ True and $h$ False are the elements represented in Figure 6 . For $x \neq$ True and $x \neq$ False, we define $h x=x$. It can easily be seen that the operations are well defined and $A X$ satisfies the conditions in Definition 3.3.

For the sake of convenience, we recall some definitions in [13]. 
Definition 3.4. For any $h, k \in L H$, we shall write $h x<\leq k x(h x<\leq I x)$ if for any $h^{\prime}, k^{\prime}$ in $U O S$ and any $m, n \in N a t, V^{n} h^{\prime} h x \leq V^{m} k^{\prime} k x\left(V^{n} h^{\prime} h x \leq I x\right)$. If the last inequalities are always strict, then we shall write $h x<<k x(h x<<I x)$.

As an example, the inequality $V^{n}$ Very More true $\leq V^{m}$ Little Very true holds intuitively, for all $n$ and $m$ and so we can write More true $<\leq$ Very true.

Definition 3.5. Let $x$ and $u$ be two elements in an RHA $A X=(X, G, L H, \leq)$. The expression $h_{n} \ldots h_{1} u$ is said to be a canonical representation of $x$ w.r.t. $u$ in $A X$ if

(i) $x=h_{n} \ldots h_{1} u$; (ii) $h_{i} \ldots h_{1} u \neq h_{i-1} \ldots h_{1} u$ for every $i \leq n$.

Theorem 3.1. Let $A X=(X, G, L H, \leq)$ be an RHA. Then, the following statements hold:

(o) If $h x<\leq k x$ then $h x \leq k x$.

(i) The operations in $L H^{c}$ are compatible.

(ii) If $x \in X$ is a fixed point of an operation $h$ in $L H$, i.e. $h x=x$, then it is a fixed point of the other ones.

(iii) If $x=h_{n} \ldots h_{1} u$, then there exists an index $i$ such that the suffix $h_{i} \ldots h_{1} u$ of $x$ is a canonical representation of $x$ w.r.t. $u$ and $h_{j} x=x$, for all $j>i$.

(iv) If $h \neq k$ and $h x=k x$ then $x$ is a fixed point.

(v) For any $h, k \in L H$, if $x \leq h x(x \geq h x)$ then $I x<\leq h x(I x \geq>h x)$ and if $h x \leq k x, h \neq k$ and there is no $i$ in $S I^{c}$ such that both $h$ and $k$ belong to $L H_{i}^{c}$, then $h x<\leq k x$.

Proof. First, we observe that the condition (R1) is the same as the axioms (A1) and (A2) in Definition 3[13]. Therefore, the proofs of (o), (i), (ii), (iii) which are based on (R1) are similar as that in [13].

By (ii), we can use the terminology "a fixed point" instead of "a fixed point of an operation".

Now we prove (iv). Assume the contrary, that $x$ is not a fixed point. Suppose that $h x>x$. If $h$ and $k$ are converse the $k x \leq x$. Hence, $h x>x \geq k x$, which contradicts the hypothesis. If $h$ and $k$ are compatible then on account of Definition 3.2 and $h \neq k$, it follows that $h x \neq k x$ if $h x \neq x$, which is again impossible. Thus $h x=x$. For the case where $h x<x$, the proof is similar. This concludes the proof of (iv).

To prove $(\mathrm{v})$, suppose that $x \leq h x$. If $h x=x$ then $x$ is a fixed point and so $x \leq$ $V^{n} h^{\prime} h x$, for each $h^{\prime} \in U O S$. If $h x>x$, by virtue of (R2), we have $x \notin L H(h x)$ and $x \leq h^{\prime} h x$, for $h^{\prime} \in U O S$, by (R4). Again by (R4) we obtain $x \leq V h^{\prime} h x$. Since $x \in L H(x)$ and $x \notin L H(h x)$, applying (R4) repeatedly, we have $x \leq V^{n} h^{\prime} h x$, i.e. $I x<\leq h x$, by Definition 3.4.

For the case where $x \geq h x$, the proof is similar.

Now suppose that $h x \leq k x, h \neq k$ and $h$ and $k$ do not together belong to $L H_{i}^{c}$ for any $i$. If $h x=k x$ then $x$ is a fixed point, by (iv) of the theorem. Thus, $V^{n} h^{\prime} h x=V^{m} k^{\prime} k x$, for all $h^{\prime}, k^{\prime} \in U O S$ and $m, n \in$ Nat.

Assume that $h x<k x$ and $k \in L H_{i_{0}}^{c}$, and $h \in L H_{i_{1}}^{c}$, for $i_{1}, i_{0} \in I^{c}$ and $i_{1} \neq i_{0}$. Assuming that $h$ and $k$ are converse, we have $h x<x<k x$. As proved above, it follows that $h x<<x$ and $x<<k x$. Thus $h x<<k x$. Now assume that $h$ and $k$ are compatible. 
Put $u=h x$. Since $h x \neq k x$, it follows from (iv) of the theorem that $h x \neq k_{1} x$ for any $k_{1} \in L H_{i_{0}}^{c}$. By (R3), $h x$ and $k_{1} x$ are independent and $u=h x \notin L H\left(k_{1} x\right)$, for any $k_{1} \in L H_{i_{0}}^{c}$. Since $u \in L H(x)$, it follows from (R4) that $u<k^{\prime} k x$, for any $k^{\prime} \in U O S$. Applying (R4) again to the last inequality, we get $u<V k^{\prime} k x$. Repeating this argument, it implies that $u=h x<V^{m} k^{\prime} k x$, for any $m \in N a t$ and $k^{\prime} \in U O S$. It can be seen that $h$ and $k$ play a similar role and hence we can use the analogous argument as above, where $u=V^{m} k^{\prime} k x$, and we obtain $V^{m} k^{\prime} k x>V^{n} h^{\prime} h x$, for any $h^{\prime}, k^{\prime} \in U O S$ and $m, n \in$ Nat, which means that $h x<<k x$. So, we have proved that $h x<\leq k x$, which completes the proof of (v).

The following theorem is a reformulation of Theorem 2 in [13] for RHAs.

Theorem 3.2. For any $h \in L H$, there exist two unit operations $h^{-}$and $h^{+}$such that $h^{-}$is negative and $h^{+}$is positive w.r.t. $h$ and for any $h_{1}, \ldots, h_{n} \in L H, x \in X$,

$$
\begin{aligned}
& V^{n} h^{-} h x \leq h_{n} \ldots h_{1} h x \leq V^{n} h^{+} h x \quad \text { if } h x \geq x, \\
& V^{n} h^{-} h x \geq h_{n} \ldots h_{1} h x \geq V^{n} h^{+} h x \quad \text { if } h x \leq x .
\end{aligned}
$$

Proof. We shall prove the theorem by induction on the number $n$ of hedge operations. Assume $n=1$ and $h x \geq x$. If $h_{1}$ is positive w.r.t. $h$ then we have $h_{1} h x \geq h x$. From the assumptions of the operations $h^{+}$and $h^{-}$, we have $h^{+} h x \geq h_{1} h x \geq h x \geq h^{-} h x$. Since $V$ is positive w.r.t. $h^{+}$and $h^{-}$(see [13]), we obtain $V h^{+} h x \geq h^{+} h x \geq h_{1} h x \geq h x \geq$ $h^{-} h x \geq V h^{-} h x$, which are the required inequalities.

If $h_{1}$ is negative w.r.t. $h$, we have $h_{1} h x \leq h x$. By the assumption on the hedge $h^{-}$, it follows that $h^{-}$and $h_{1}$ are compatible and $h^{-} \geq h_{1}$. So $h^{-} h x \leq h_{1} h x \leq h x$. By the same argument as above, we obtain again $V h^{+} h x \geq h^{+} h x \geq h x \geq h_{1} h x \geq h^{-} h x \geq V h^{-} h x$.

For the case $h x \leq x$, the proof is similar. Consequently, it has been proved that the inequalities in the theorem hold for $n=1$.

Assume that the theorem holds for $n=i$, i.e. if $h_{1} h x \leq h x$, then $V^{i} h_{1}^{+} h_{1} h x \leq$ $h_{i+1} h_{i} \ldots h_{1} h x \leq V^{i} h_{1}^{-} h_{1} x$ and if $h_{1} h x \geq h x$ then $V^{i} h_{1}^{+} h_{1} h x \geq h_{i+1} h_{i} \ldots h_{1} h x \geq$ $V^{i} h_{1}^{-} h_{1} x$, where $h_{1}^{+}, h_{1}^{-}$and $h_{1}$ satisfy the assumption like that made on $h^{+}, h^{-}$and $h$.

Now we shall prove the induction conclusion for the case $h x \leq x$. For the opposite case, the proof is similar.

Suppose first that $h_{1}$ is positive w.r.t. $h$, and so $h_{1} h x \leq h x$. From the induction hypothesis it follows that $h_{i+1} h_{i} \ldots h_{1} h x \leq V^{i} h_{1}^{-} h_{1} h x$, and by (v) of Theorem 3.1, it implies that $V^{i} h_{1}^{-} h_{1} h x \leq h x \leq h^{-} h x \leq V^{i+1} h^{-} h x$, with a notice that $h^{-}$is negative w.r.t. $h$ and $V$ is positive w.r.t. $h^{-}$and $V$. So, one of the two required inequalities is true.

Since both $h_{1}$ and $h^{+}$are positive w.r.t. $h$, it follows that they together belong to either $L H^{+}$or $L H^{-}$. So, $h^{+} \geq h_{1}$ and hence $h^{+} h x \leq h_{1} h x$. In addition, if $h^{+} \neq h_{1}$, then by (v) of Theorem 3.1, it follows that $h^{+} h x<\leq h_{1} h x$. According to the induction hypothesis and Definition 3.4, we have $h_{i+1} h_{i} \ldots h_{1} h x \geq V^{i} h_{1}^{+} h_{1} h x \geq V^{i} V h^{+} h x$. If $h^{+}=h_{1}$, then $h_{1}$ is either $V$ or $L$. In both cases $h^{+}=V$ and, hence, from the induction hypothesis it follows that $h_{i+1} h_{i} \ldots h_{1} h x \geq V^{i} V h_{1} h x=V^{i+1} h^{+} h x$. Thus, for the case where $h_{1}$ is positive w.r.t. $h$, the induction conclusion follows. Since the proof for the case where $h_{1}$ is negative w.r.t. $h$ is similar, the theorem is completely proved. 
Corollary 3.1. (i) For any $x \in X$, if $h x<k x$ and there is no $i \in S I^{c}$ such that both $h$ and $k$ belong to $L H_{i}^{c}$, then for any two strings of hedges $\delta$ and $\delta^{\prime}$, the inequality $\delta h x<\delta^{\prime} k x$ holds.

(ii) Let $u$ be an arbitrary element in $X$ and $x \in L H(u)$. Then, there exist always elements $y, z \in U O S(u)$, i.e. $z$ and $y$ are generated from $u$ by means of the unit operations, such that $y \geq x \geq z$. Furthermore, either one of the equalities $u \leq x \leq V^{n}$ hu and $u \geq x \geq V^{n} h u$ holds, for a suitably chosen $h \in L H$ and for sufficiently great number $n \in$ Nat.

PROOF. For the proof of this corollary, we refer the reader to [13].

Now, the following theorem gives us a characterisation to determine the relative position of elements in an RHA. Here, the notation $x_{j}$ is defined as follows: if $x=h_{n} \ldots h_{1} u$, then $x_{j}$ denotes the expression $h_{j-1} \ldots h_{1} u$, for $1 \leq j \leq n$.

THEOREM 3.3. Let $x=h_{n} \ldots h_{1} u$ and $y=k_{m} \ldots k_{1} u$ be two arbitrary canonical representations of $x$ and $y$ w.r.t. $u$, respectively. Then there exists an index $j \leq \min (m, n)+1$ such that $h_{j^{\prime}}=k_{j^{\prime}}$, for all $j^{\prime}<j$ and

(1) $x<y$ iff one of the following conditions holds

(i) $h_{j} x_{j}<k_{j} x_{j}$ and $\delta k_{j} x_{j} \leq \delta^{\prime} k_{j} x_{j}$ or $\delta h_{j} x_{j} \leq \delta^{\prime} h_{j} x_{j}$ if $h_{j}$ and $k_{j}$ together belong to $L H_{i}^{c}$ for some $i \in S I^{c}$, where $x_{j}=h_{j-1} \ldots h_{1} u, \delta=h_{n} \ldots h_{j+1}, \delta^{\prime}=$ $k_{m} \ldots k_{j+1}$;

(ii) $h_{j} x_{j}<k_{j} x_{j}$, otherwise;

(2) $x=y$ iff $m=n$ and $h_{j}=k_{j}$ for all $j \leq n$;

(3) $x$ and $y$ are incomparable iff there exists $i \in S I^{c}$ such that both $h_{j}$ and $k_{j}$ belong to $L H_{i}^{c}$ and one of the following conditions holds:

(i) $h_{j} x_{j}$ and $k_{j} x_{j}$ are incomparable,

(ii) $h_{j} x_{j}<k_{j} x_{j}$ and $\delta k_{j} x_{j} \not \leq \delta^{\prime} k_{j} x_{j}$,

(iii) $h_{j} x_{j}>k_{j} x_{j}$ and $\delta^{\prime} h_{j} x_{j} \not \leq \delta h_{j} x_{j}$.

Proof. Let $j$ be the least index such that $h_{j} \neq k_{j}$. It can be seen that $j \leq \min (m, n)+$ 1 , since $I \neq h$ for every $h \in L H$.

Sufficiency: To prove the sufficiency of (1), suppose first that $h_{j} x_{j}<k_{j} x_{j}$ and there is no index $i_{0}$ in $S I^{c}$ such that both $h_{j}$ and $k_{j}$ belong to $L H_{i_{0}}^{c}$. From (v) of Theorem 3.1, we obtain $h_{j} x_{j}<<k_{j} x_{j}$ and $V^{p} h h_{j} x_{j}<V^{q} k k_{j} x_{j}$, for any $h, k \in U O S$ and $p, q \in$ Nat. By Theorem 3.2, there exist $h^{\prime}, k^{\prime} \in U O S$ such that $h_{n} \ldots h_{j} x_{j} \leq V^{n-j-1} h^{\prime} h_{j} x_{j}$ and $k_{m} \ldots k_{j} x_{j} \geq V^{m-j-1} k^{\prime} k_{j} x_{j}$, which imply that $x<y$.

If there exists an index $i_{0}$ in $S I^{c}$, such that both $h_{j}$ and $k_{j}$ belong to $L H_{i_{0}}^{c}$, and $h_{j} x_{j}<k_{j} x_{j}$ and $\delta k_{j} x_{j} \leq \delta^{\prime} k_{j} x_{j}$, then by (R3), we have $\delta h_{j} x_{j}<\delta k_{j} x_{j}$. Hence, $x=$ $\delta h_{j} x_{j}<\delta k_{j} x_{j} \leq \delta^{\prime} k_{j} x_{j}=y$.

Since the sufficiency of (2) is evident, we prove the sufficiency of (3). Suppose that there exists an index $i_{0}$ in $S I^{c}$ such that both $h_{j}$ and $k_{j}$ belong to $L H_{i_{0}}^{c}$. If (i) holds, it follows from (R3) that $x$ and $y$ are incomparable. If (ii) holds, i.e. $h_{j} x_{j}<k_{j} x_{j}$ and $\delta k_{j} x_{j} \not \subset \delta^{\prime} k_{j} x_{j}$, then by (R3), we infer $\delta h_{j} x_{j}<\delta k_{j} x_{j}$. Moreover, it follows from (R3) that $\delta h_{j} x_{j}$ and $z$ are incomparable, for any $z \in L H\left(k_{j} x_{j}\right)$ such that $\delta k_{j} x_{j} \not \leq z$. Thus, $x=\delta h_{j} x_{j}$ and $y=\delta^{\prime} k_{j} x_{j}$ are incomparable. In the case (iii) holds, the proof is similar. 
Necessity: Suppose that there is no index $j$ such that $h_{j} \neq k_{j}$. Note that one of $h_{j}$ and $k_{j}$ may be the operation $I$. Then, it is evident that the two canonical representations of $x$ and $y$ are identical and hence $x=y$. Therefore, assuming that these two canonical representations are different, there exists the least index $j$ such that $h_{j} \neq k_{j}$. Obviously, $j \leq \min (m, n)+1$. Between $h_{j} x_{j}$ and $k_{j} x_{j}$ there are the following ordering relationships: $h_{j} x_{j}=k_{j} x_{j}, h_{j} x_{j}<k_{j} x_{j}, h_{j} x_{j}>k_{j} x_{j}$ and $h_{j} x_{j}$ and $k_{j} x_{j}$ are incomparable. From the proof of the sufficiency, we have the following:

(1) If $x<y$ then $h_{j} x_{j}<k_{j} x_{j}$. Furthermore, if there exists $i_{0}$ in $S I^{c}$ such that both $h_{j}$ and $k_{j}$ belong to $L H_{i_{0}}^{c}$, then, by (R3), from $\delta k_{j} x_{j} \not \leq \delta^{\prime} k_{j} x_{j}$ it follows that $x$ and $y$ are incomparable. This contradicts the hypothesis and hence, $\delta k_{j} x_{j} \leq \delta^{\prime} k_{j} x_{j}$. Likewise, it can be proved that $\delta h_{j} x_{j} \leq \delta^{\prime} h_{j} x_{j}$.

(2) If $x=y$ then $h_{j} x_{j}=k_{j} x_{j}$. It remains to prove that if $h_{j} x_{j}=k_{j} x_{j}$ then $m=n$ and $h_{j}=k_{j}$. In fact, if $h_{j} \neq k_{j}$, it follows by (iv) of Theorem 3.1 that $x_{j}$ is a fixed point. Thus, from the definition of the canonical representations it follows that $m=n$ and $h_{j}=k_{j}$ for all $j \leq n$.

(3) Suppose that $x$ and $y$ are incomparable. Then, there are only three possibilities: $h_{j} x_{j}<k_{j} x_{j}, h_{j} x_{j}>k_{j} x_{j}$ and $h_{j} x_{j}$ and $k_{j} x_{j}$ are incomparable. If $h_{j}$ and $k_{j}$ are converse then it is easy to infer that $x$ and $y$ are comparable, a contradiction. If $h_{j}$ and $k_{j}$ are compatible and there is no $i_{0}$ in $S I^{c}$ such that both $h_{j}$ and $k_{j}$ belong to $L H_{i_{0}}^{c}$, then from Remark 3.1, it follows that $h_{j}$ and $k_{j}$ are comparable. So, $h_{j} x_{j}$ and $k_{j} x_{j}$ are also comparable. Furthermore, from (v) of Theorem 3.1 and (ii) of Corollary 3.1, it is easy to check that $x$ and $y$ are comparable, as well. This contradicts the assumption. Thus, we have proved that if $x$ and $y$ are incomparable, then there exists an $i_{0}$ in $S I^{c}$ such that both $h_{j}$ and $k_{j}$ belong to $L H_{i_{0}}^{c}$. Assume now that $h_{j} x_{j}<k_{j} x_{j}$. We have to prove that $\delta k_{j} x_{j} \not \leq \delta^{\prime} k_{j} x_{j}$. In fact, by (R3), we have $\delta h_{j} x_{j}<\delta k_{j} x_{j}$. If $\delta k_{j} x_{j} \leq \delta^{\prime} k_{j} x_{j}$ then $x=\delta h_{j} x_{j}<\delta k_{j} x_{j} \leq \delta^{\prime} k_{j} x_{j}=y$, which contradicts the hypothesis. Thus, we have $\delta k_{j} x_{j} \not \leq \delta^{\prime} k_{j} x_{j}$. In the case $h_{j} x_{j}>k_{j} x_{j}$, by an analogous argument we have $\delta^{\prime} h_{j} x_{j} \not \leq$ $\delta h_{j} x_{j}$. This concludes the proof.

REMARK 3.2. At first glance, one may think that the theorem is meaningless, because it replaces the comparison of two elements by the comparison of two others: The comparison between $x=\delta h_{j} x_{j}$ and $y=\delta^{\prime} k_{j} x_{j}$ is changed to that between $x^{\prime}=\delta k_{j} x_{j}$ and $y^{\prime}=\delta^{\prime} k_{j} x_{j}$ or between $x^{\prime}=\delta h_{j} x_{j}$ and $y^{\prime}=\delta^{\prime} h_{j} x_{j}$. But, notice that the length of the common suffix of $x^{\prime}$ and $y^{\prime}$ is greater than that of $x$ and $y$. It leads to a procedure that with a finite number of steps one can decide whether the given elements $x$ and $y$ are comparable and which one is greater than the other.

Corollary 3.2. If $x$ is not a fixed point and $u$ is any element in $X$, then the canonical representation of $x$ w.r.t. $u$, if it exists, is unique, i.e. if $h_{n} \ldots h_{1} u$ and $k_{m} \ldots k_{1} u$ are two canonical representations of $x$ w.r. $t$. $u$, then $m=n$ and $h_{i}=k_{i}$, for all $i \leq n$.

The following proposition shows that if both $h$ and $k$ belong to $L H_{i}^{c}$, for $i \in S I^{c}$, then from the property $h x$ is a fixed point we can deduce that $k x$ is also fixed point and vice-versa. Intuitively, it means that, for such $h$ and $k, h$ can generate a proper meaning from an element $x$ (i.e. $h x \neq x$ ) iff $k$ does so. 
Proposition 3.1. For any $x \in X$ and $i \in S I^{c}$. If there exists a hedge $h \in L H_{i}^{c}$ such that hx is a fixed point, then so is $k x$, for any $k \in L H_{i}^{c}$.

Proof. If $h x=k x$, then the assertion is evident. Assume that $h x \neq k x$. We shall prove by cases as follows:

(i) Assume that $h x<k x$. It follows that $V h x<V k x$ and if $V k x>k x$, then $k x$ and $V h x$ are incomparable, by (i) of (R3). It contradicts the assumption that $V h x=h x<k x$. If $V k x<k x$, then $V k x$ and $h x$ are incomparable, by (i) of (R3) and this again contradicts the fact that $h x=V h x<V k x$. Since, by (R1), $V k x$ and $k x$ must be comparable, it follows that $V k x=k x$, i.e. $k x$ is a fixed point.

(ii) For the case where $h x>k x$, the proof is similar.

(iii) Suppose that $h x$ and $k x$ are incomparable. Since $L H_{i}^{c}$, for $i \in S I^{c}$, is a sublattice of $L H^{c}+I$, it follows that $(h \vee k)$ belongs to $L H_{i}^{c}$. Clearly, $h x$ and $(h \vee k) x$ are comparable. By the cases proved above, it follows that $(h \vee k) x$ is a fixed point and, hence, by the same reason, $k x$ is a fixed point. The proof is completed.

The following proposition can be considered as a generalization of Proposition 3.1.

Proposition 3.2. For any $x \in X$ and $h, k \in L H_{i}^{c}$, for some $i \in S I^{c}$ and for any string of hedges $\delta, \delta h x$ is a fixed point iff $\delta k x$ is a fixed point.

Proof. Suppose that $\delta h x$ is a fixed point. There are two cases:

Case (i): $h x$ and $k x$ are comparable. Without loss of generality, suppose that $h x \leq k x$. If $h x=k x$ then, by (iv) of Theorem $3.1, x$ is a fixed point and, hence, so is $\delta k x=x$. Now, assume that $h x<k x$. By (i) of (R3), it follows that $\delta h x<\delta k x$.

Suppose the contrary that $\delta k x$ is not a fixed point. Take a suitable $h^{\prime}$ so that $h^{\prime} \delta k x<$ $\delta k x$. By (i) of (R3), $\delta h x$ and $h^{\prime} \delta k x$ are incomparable. Again by (i)(R3), from $h x<k x$ it follows that $\delta h x=h^{\prime} \delta h x<h^{\prime} \delta k x$. We have a contradiction. Therefore, $\delta k x$ is a fixed point.

Case (ii): $h x$ and $k x$ are incomparable. By the same argument as in Case (iii) of the proof of Proposition 3.1, we can prove that $\delta k x$ is a fixed point.

Since $h$ and $k$ play symmetrical roles, the proof is completed.

Since the RHA is constructed from a given PN-homogeneous hedge algebra, a natural question arises whether the PN-homogeneous property for the unit-operation $\mathrm{V}$ in $\mathrm{LH}^{+}+$ $I$, but not in $H^{+}+I$, still holds if we replace $H_{i}^{c}$ in Definition 3.1 with $L H_{i}^{c}$. The following proposition answers this question.

Proposition 3.3. If the unit operation $\mathrm{V}$ in $\mathrm{LH}^{+}+\mathrm{I}$ is positive (negative, resp.) w.r.t. a certain $h$ in $H_{i}^{c}$, for $i$ in $S I^{c}$, then $V$ is also positive (negative, resp.) w.r.t. any operation in $L_{i}^{c}$.

PRoOF. We shall prove the assertion for the case of "positive". The proof for the case of "negative" is similar.

Assume that $V$ is positive w.r.t. $h \in H_{i}^{c}$, for some $i \in S I^{c}$. Since the hedge algebra $(H(G), G, H, \leq)$ is PN-homogeneous, it follows that $V$ is also positive w.r.t. any operation in $H_{i}^{c}$. Since $L H_{i}^{c}$ is a free distributive lattice generated from incomparable elements in $H_{i}^{c}$, for every $k \in L H_{i}^{c}$ there exists $h^{\prime} \in H_{i}^{c}$ such that either $k \geq h^{\prime}$ or $k \leq h^{\prime}$. If $k=h^{\prime}$, 
then $V$ is already positive w.r.t. $k$. Consider the case $h^{\prime} \neq k$ and suppose that $h^{\prime} x \leq k x$. In the case $h^{\prime} x=k x$, by (iv) of Theorem 3.1, $x$ is a fixed point and so $V k x=k x$. Assume that $h^{\prime} x<k x$, we have $V h^{\prime} x<V k x$, by (i)(R3). If $h^{\prime} x$ is a fixed point, then so is $k x$, by Proposition 3.1, i.e. $V k x=k x$.

If $V h^{\prime} x>h^{\prime} x$, then $h^{\prime} x>x$, since $V$ is positive w.r.t. $h^{\prime}$. Hence, $k x \geq x$ and, again by (i)(R3) with $\delta$ to be empty, $k x$ and $V h x$ are incomparable. From this fact and the inequality $V h^{\prime} x<V k x$ it follows from (i)(R3) that $V k x \geq k x \geq x$.

If $V h^{\prime} x<h^{\prime} x$, then $h^{\prime} x<x$, since $V$ is positive w.r.t. $h^{\prime}$. From (i)(R3), it follows that $h^{\prime} x$ and $V k x$ are incomparable and, similarly as above, it can be derived that $V k x \leq$ $k x \leq x$.

Since an analogous argument can be used for the case $h^{\prime} x \geq k x$, we have proved that, in any case, either $V k x \leq k x \leq x$ or $V k x \geq k x \geq x$, i.e. $V$ is positive w.r.t. $k$. This concludes the proof.

The following proposition states that hedge operations in the same sublattice have analogous semantic properties in terms of $\leq$.

Proposition 3.4. For any $h, k \in L H_{i}^{c}$, for some $i \in S I^{c}$, and for any $x \in X$, we have the following assertions:

(i) $\delta h x>x(\delta h x<x)$ iff $\delta k x>x(\delta k x<x)$, for any $\delta \in L H^{*}$.

(ii) If $h x \neq k x$, then $\delta h x$ and $\delta^{\prime} h x$ are incomparable iff $\delta k x$ and $\delta^{\prime} k x$ are incomparable, for any $\delta, \delta^{\prime} \in L H^{*}$.

(iii) $\delta h x>\delta^{\prime} h x$ iff $\delta k x>\delta^{\prime} k x$, for any $\delta, \delta^{\prime} \in L H^{*}$.

Proof. The assertion (i) can easily be proved from the given assumption on $h$ and $k$.

(ii) It is sufficient to prove the statement for the case where $h$ and $k$ are comparable, since if $h$ and $k$ are incomparable in $L H_{i}^{c}$, then there exists $h^{\prime}$ in $L H_{i}^{c}$ such that $h^{\prime} \geq k$ and $h^{\prime} \geq h$. Hence, it can easily be seen that the assertion can be deduced from the case being proved now. Moreover, without loss of generality, we can assume that $h x>k x$. For any two strings of hedges $\delta$ and $\delta^{\prime}$, it follows from (i)(R3) that $\delta h x>\delta k x$ and $\delta^{\prime} h x>\delta^{\prime} k x$, and that the incomparability of $\delta h x$ and $\delta^{\prime} h x$ implies the incomparability of two elements $\delta^{\prime} h x$ and $\delta k x$ and that of two elements $\delta h x$ and $\delta^{\prime} k x$. Now, it can be verified that the comparability of $\delta k x$ and $\delta^{\prime} k x$ leads to a contradiction. Similarly, we can prove that the incomparability of $\delta k x$ and $\delta^{\prime} k x$ implies the incomparability of $\delta h x$ and $\delta h x$.

(iii) Similar as in the proof of (ii), we can assume without loss of generality that $h x>k x$. It follows from (R3) that $\delta h x>\delta k x$ and $\delta^{\prime} h x>\delta^{\prime} k x$, for any $\delta, \delta^{\prime} \in L H^{*}$. Suppose now that $\delta h x>\delta^{\prime} h x$. It implies from (ii) that $\delta k x$ and $\delta^{\prime} k x$ are comparable. Further, by (R3), we infer that $\delta k x$ and $\delta^{\prime} h x$ are incomparable and so, if $\delta k x \leq \delta^{\prime} k x$ then $\delta k x<\delta^{\prime} h x$, we have a contradiction. Hence, $\delta k x>\delta^{\prime} k x$. Since the sufficiency is evident, the proof is completed.

4. Lattice characteristic and distributivity of RHAs. In this section, we shall study the main property of RHAs. It will be shown that RHA is a distributive lattice if the set of the primary generators is a chain. Firstly, we shall prove the following theorem 
saying that RHA with a chain of the primary generators is a lattice. It also gives us recursive formulas for computing infimum and supremum of elements in RHA.

TheOREM 4.1. Let $A X=(X, G, L H, \leq)$ be an $R H A$ and $G$ be a chain of generators. Then $A X$ is a lattice. Moreover, for any two incomparable elements $x$ and $y$ in $X$, if $x=\delta h w$, and $y=\delta^{\prime} k w$, where $\delta, \delta^{\prime} \in L H^{*}$ and $w \in L H(a)$ for some $a \in G$, are canonical representations of $x$ and $y$, respectively, then both $h$ and $k$ belong to $L H_{i}^{c}$, for some $i \in S I^{c}$ and

$$
\begin{aligned}
& x \cup y=\left\{\begin{array}{l}
\delta(h \vee k) w \cup \delta(h \vee k) w \text { if } h w>w \\
\delta(h \wedge k) w \cup \delta(h \wedge k) w \text { if } h w<w
\end{array}\right. \\
& x \cap y=\left\{\begin{array}{l}
\delta(h \wedge k) w \cup \delta(h \wedge k) w \text { if } h w>w \\
\delta(h \vee k) w \cup \delta(h \vee k) w \text { if } h w<w
\end{array}\right.
\end{aligned}
$$

where $\cup, \cap$ stand for join, meet in $A X$, while $\vee, \wedge$ stand for join and meet in $L H^{c}+I$.

Proof. From (R2) it follows that if $x$ and $y$ are incomparable in $X$, then there exists an element $a \in G$ such that $x, y \in L H(a)$, since $G$ is a chain. Thus, there exist two canonical representations of $x$ and $y$ w.r.t. $a$, say $x=h_{n} \ldots h_{1} a$ and $y=k_{m} \ldots k_{1} a$. On account of Theorem 3.3, there exists an index $j \leq \min (m, n)+1$ such that $h_{i}=k_{i}$, for any $i \leq j$. Furthermore, there exists $i_{0} \in S I^{c}$ such that $h_{j}, k_{j} \in L H_{i_{0}}^{c}$. Let $\delta=h_{n} \ldots h_{j+1}$, $\delta^{\prime}=k_{m} \ldots k_{j+1}, h=h_{j}, k=k_{j}$. With this notation we have $x=\delta h w$ and $y=\delta^{\prime} k w$, where $w=h_{j-1} \ldots h_{1} a$.

We shall prove the theorem for the supremum. The proof for the infimum can be obtained by duality.

Let us first consider the case where $h w>w$. Then, we also have $k w>w$. It implies that $(h \vee k) w>w$ and $h \vee k \in L H_{i_{0}}^{c}$, since $L H_{i_{0}}^{c}$ is a sublattice of $L H^{c}+I$. By Definition 3.2 and (i)(R3), we have $\delta(h \vee k) w \geq \delta h w, \delta^{\prime}(h \vee k) w \geq \delta^{\prime} k w$.

We shall prove that $t \geq\left\{\delta(h \vee k) w, \delta^{\prime}(h \vee k) w\right\}$, for any $t \in L H(a), t>\{x, y\}$.

Suppose that $t=l_{p} \ldots l_{1} a$ is the canonical representation of $t$ w.r.t. $a$. Consider the case that $t \in L H(w)$ and so we have $t=l_{p} \ldots l_{j+1} l_{j} w$. Since $t>\{x, y\}$ it follows from Theorem 3.3 that $l_{j} w>\{h w, k w\}$. Remember that $h w>w$ and, hence, $l_{j} \geq h \vee k$ and $l_{j} w \geq(h \vee k) w$. If $l_{i} \notin L H_{i_{0}}^{c}$, by (v) Theorem 3.1 we obtain $l_{j} w>>(h \vee k) w$ and so, we can infer that

$$
t>\left\{\delta(h \vee k) w, \delta^{\prime}(h \vee k) w\right\} .
$$

Let $l_{j} \in L H_{i_{0}}^{c}$ and assume that $l_{j} w=(h \vee k) w$. If $l_{j} \neq(h \vee k)$, then by (iv) Theorem $3.1, w$ is a fixed point and hence $w=h w$, contrary to assumption. Thus, $l_{j}=(h \vee k)$ and, since $l_{j} w>h w, l_{j} w>k w$ and $t>\{x, y\}$, Theorem 3.3 yields

$$
t \geq\left\{\delta(h \vee k) w, \delta^{\prime}(h \vee k) w\right\} .
$$

Now, assume that $l_{j} w>(h \vee k) w$. Thus, $t=l_{p} \ldots l_{j} w>l_{p} \ldots l_{j+1}(h \vee k) w$, by (i)(R3). Since $t>\{x, y\}$ and it is easily seen that $x=\delta h w<\delta l_{j} w, y=\delta^{\prime} k w<\delta^{\prime} l_{j} w$, we infer again by (i)(R3) that $t \geq\left\{\delta l_{j} w, \delta^{\prime} l_{j} w\right\}$. Applying Proposition 3.4 to the last inequalities, we get

$$
l_{p} \ldots l_{j+1}(h \vee k) w \geq\left\{\delta(h \vee k) w, \delta^{\prime}(h \vee k) w\right\} .
$$

Hence, $t>\left\{\delta(h \vee k) w, \delta^{\prime}(h \vee k) w\right\}$, which is the desired inequality. 
Now, consider the case that $t \notin L H(w)$. Then, there exists an index $j^{\prime} \leq j-1$ such that $h_{i}=l_{i}$ for any $i<j^{\prime}$ and $l_{j^{\prime}} u>h_{j^{\prime}} u$, where $u=h_{j^{\prime}-1} \ldots h_{1} a$. If there is no $i_{1} \in S I^{c}$ such that $h_{j^{\prime}}, l_{j^{\prime}} \in L H_{i_{1}}^{c}$ then it follows from (v) Theorem 3.1 that $l_{j^{\prime}} u>>h_{j^{\prime}} u$ and, hence, by Theorem 3.2, it can easily be verified that $t>\left\{\delta(h \vee k) w, \delta^{\prime}(h \vee k) w\right\}$. If there exists $i_{1} \in S I^{c}$ such that $h_{j^{\prime}}, l_{j^{\prime}} \in L H_{i_{1}}^{c}$, we set $s=j-j^{\prime}-1$ and prove the assertion by induction on the number $s$ of hedge operations.

For $s=0$, i.e. $j^{\prime}=j-1$, and $w=h_{j^{\prime}} u$, we can write $t \geq\left\{x=\delta h h_{j^{\prime}} u, y=\delta^{\prime} k h_{j^{\prime}} u\right\}$, and applying (i)(R3) to $l_{j^{\prime}} u>h_{j^{\prime}} u$, it follows that $t \geq\left\{\delta h l_{j^{\prime}} u, \delta^{\prime} k l_{j^{\prime}} u\right\}$. Since $h h_{j^{\prime}} u>$ $h_{j^{\prime}} u$ and the elements $x=\delta h h_{j^{\prime}} u$ and $y=\delta^{\prime} k h_{j^{\prime}} u$ are incomparable, it follows also from Proposition 3.4 that $h l_{j^{\prime}} u>l_{j^{\prime}} u$ and that $\delta h l_{j^{\prime}} u$ and $\delta^{\prime} k l_{j^{\prime}} u$ are incomparable. Clearly, $t \in L H\left(l_{j^{\prime}} u\right)$ and analogously to the case where $t \in L H(w)$, with $w=l_{j^{\prime}} u$, we can prove that $t \geq\left\{\delta(h \vee k) l_{j^{\prime}} u, \delta^{\prime}(h \vee k) l_{j^{\prime}} u\right\}$. Moreover, it follows from (i)(R3) that $\delta(h \vee k) l_{j^{\prime}} u>\delta(h \vee k) h_{j^{\prime}} u=\delta(h \vee k) w$ and $\delta^{\prime}(h \vee k) l_{j^{\prime}} u>\delta^{\prime}(h \vee k) h_{j^{\prime}} u=\delta^{\prime}(h \vee k) w$ and, hence, $t>\left\{\delta(h \vee k) w, \delta^{\prime}(h \vee k) w\right\}$.

Assume the induction hypothesis, that the inequality holds for every $s \leq i$. For $s=$ $i+1$, we have $j^{\prime}+i+1=j-1$ and $w=h_{j-1} \ldots h_{j^{\prime}+1} h_{j^{\prime}} u$. Set $w^{\prime}=h_{j-1} \ldots h_{j^{\prime}+1} l_{j^{\prime}} u$. It follows from Proposition 3.4 that $h w^{\prime}>w^{\prime}$, since $h w>w$ and $h_{j^{\prime}}, l_{j^{\prime}} \in L H_{i_{1}}^{c}$. Using again (i)(R3) as above, we get

$$
t \geq\left\{\delta h h_{j-1} \ldots h_{j^{\prime}+1} l_{j^{\prime}} u, \delta^{\prime} k h_{j-1} \ldots h_{j^{\prime}+1} l_{j^{\prime}} u\right\}
$$

and by Proposition 3.4 we see that $\delta h h_{j-1} \ldots h_{j^{\prime}+1} l_{j^{\prime}} u$ and $\delta^{\prime} k h_{j-1} \ldots h_{j^{\prime}+1} l_{j^{\prime}} u$ are incomparable. If $t \in L H\left(w^{\prime}\right)$ then, by the same argument as for the case $t \in L H(w)$, we obtain

$$
t \geq\left\{\delta(h \vee k) h_{j-1} \ldots h_{j^{\prime}+1} l_{j^{\prime}} u, \delta^{\prime}(h \vee k) h_{j-1} \ldots h_{j^{\prime}+1} l_{j^{\prime}} u\right\}
$$

and, hence, $t>\left\{\delta(h \vee k) w, \delta^{\prime}(h \vee k) w\right\}$, on account of (i)(R3) applied to $h_{j^{\prime}} u<l_{j^{\prime}} u$.

If $t \notin L H\left(w^{\prime}\right)$ then there exists an index $j^{\prime \prime}$, which satisfies $j^{\prime}+1 \leq j^{\prime \prime} \leq j-1$, such that $h_{i^{\prime}}=l_{i^{\prime}}$ for any $i^{\prime}$ satisfying $j^{\prime \prime}>i^{\prime} \geq j^{\prime}+1$ and $l_{j^{\prime \prime}} u^{\prime}>h_{j^{\prime \prime}} u^{\prime}$, where $u^{\prime}=l_{j^{\prime \prime}-1} \ldots l_{j^{\prime}} u$. By also an analogous argument as in the case where $t \notin L H(w)$, if there is no $i_{2} \in S I^{c}$ such that $h_{j^{\prime \prime}}, l_{j^{\prime \prime}} \in L H_{i_{2}}^{c}$ then, by the same argument as for $t \notin L H(w)$ we obtain $t>\left\{\delta(h \vee k) w^{\prime}, \delta^{\prime}(h \vee k) w^{\prime}\right\}$ and hence, $t>\left\{\delta(h \vee k) w, \delta^{\prime}(h \vee k) w\right\}$.

If there exists $i_{2} \in S I^{c}$ such that $h_{j^{\prime \prime}}, l_{j^{\prime \prime}} \in L H_{i_{2}}^{c}$ then by the induction hypothesis we have

$$
t \geq\left\{\delta(h \vee k) h_{j-1} \ldots h_{j^{\prime \prime}+1} l_{j^{\prime \prime}} u^{\prime}, \delta^{\prime}(h \vee k) h_{j-1} \ldots h_{j^{\prime \prime}+1} l_{j^{\prime \prime}} u^{\prime}\right\} .
$$

Since $h_{j^{\prime \prime}} u^{\prime}<l_{j^{\prime \prime}} u^{\prime}$, we have $\delta(h \vee k) h_{j-1} \ldots h_{j^{\prime \prime}+1} l_{j^{\prime \prime}} u^{\prime}>\delta(h \vee k) h_{j-1} \ldots h_{j^{\prime \prime}+1} h_{j^{\prime \prime}} u^{\prime}$ $>\delta(h \vee k) w$ and $\delta^{\prime}(h \vee k) h_{j-1} \ldots h_{j^{\prime \prime}+1} l_{j^{\prime \prime}} u^{\prime}>\delta^{\prime}(h \vee k) h_{j-1} \ldots h_{j^{\prime \prime}+1} h_{j^{\prime \prime}} u^{\prime}>\delta^{\prime}(h \vee k) w$, and so $t>\left\{\delta(h \vee k) w, \delta^{\prime}(h \vee k) w\right\}$, which is what we desire.

By the proved inequality, we can see that if supremum of two elements $x$ and $y$ exists then $\sup \{x, y\}=\sup \left\{\delta(h \vee k) w, \delta^{\prime}(h \vee k) w\right\}$.

So, it remains to prove that $\sup \left\{\delta(h \vee k) w, \delta^{\prime}(h \vee k) w\right\}$ always exists. Indeed, we shall argue by induction on the length of string $\delta$ of hedges. If $|\delta|=0$ then the assertion is evident, since $(h \vee k) w$ and $\delta^{\prime}(h \vee k) w$ are comparable. Assume that the assertion holds for $|\delta| \leq i$. For the case $|\delta|=i+1$, if $\delta(h \vee k) w$ and $\delta^{\prime}(h \vee k) w$ are comparable then the assertion is clearly true. If $x^{\prime}=\delta(h \vee k) w$ and $y^{\prime}=\delta^{\prime}(h \vee k) w$ are incomparable, then we 
can use the same argument as for $x$ and $y$ to prove that for any $t \in X$, if $t>\left\{x^{\prime}, y^{\prime}\right\}$ then $t \geq\left\{\delta_{1}\left(h^{\prime} \vee k^{\prime}\right) w^{\prime}, \delta_{1}^{\prime}\left(h^{\prime} \vee k^{\prime}\right) w^{\prime}\right\}$, where $h^{\prime}, k^{\prime}$ satisfy the same assumption like that on $h$ and $k$. Since $\left|\delta_{1}\right|<i, \sup \left\{\delta_{1}\left(h^{\prime} \vee k^{\prime}\right) w^{\prime}, \delta_{1}^{\prime}\left(h^{\prime} \vee k^{\prime}\right) w^{\prime}\right\}$ exists by the induction hypothesis. Consequently,

$$
\sup \left\{\delta(h \vee k) w, \delta^{\prime}(h \vee k) w\right\}=\sup \left\{\delta_{1}\left(h^{\prime} \vee k^{\prime}\right) w^{\prime}, \delta_{1}^{\prime}\left(h^{\prime} \vee k^{\prime}\right) w^{\prime}\right\}=\sup \{x, y\} .
$$

Since the proof for the case where $h w<w$ is similar, the theorem is completely proved.

For any $x \in X$, let us denote $L H[x]=\{h x / h \in L H\}$. Theorem 2.1 and Theorem 4.1 yield

Corollary 4.1. Let $A X=(X, G, L H, \leq)$ be an RHA and $G$ is a chain. The following statements hold

(i) $L H(x)$ is a sublattice of $A X$.

(ii) $L H[x]$ is a distributive sublattice of $A X$.

Proposition 4.1. Let $A X=(X, G, L H, \leq)$ be an $R H A$ and $G$ be a chain. Then, for any $h, k \in L H_{i}^{c}$, where $i \in S I^{c}$, and for any $x \in X$ such that $h x \neq k x$, there exists a lattice isomorphism $f$ from $L H(h x)$ onto $L H(k x)$ defined as follows: $f(\delta h x)=\delta k x$.

Proof. By Proposition 3.4.

Before proving the distributivity of RHA, we need the following

Theorem 4.2 [2]. Let $L$ be a lattice. $L$ is a non-distributive lattice iff $M_{5}$ or $N_{5}$ can be embedded into L, where $M_{5}$ or $N_{5}$ are two five-element lattices depicted in Figure 7 .

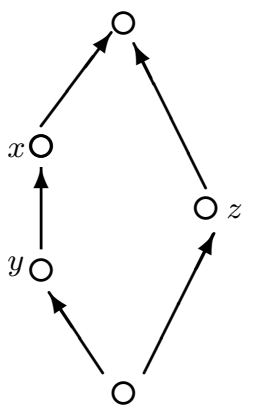

$N_{5}$

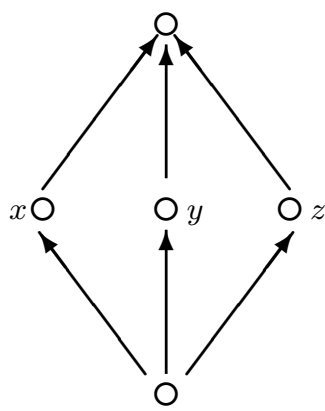

$M_{5}$

Fig. 7

Now, we shall prove the following theorem.

Theorem 4.3. Let $A X=(X, G, L H, \leq)$ be an RHA. If $G$ is a chain then $A X$ is a distributive lattice.

Proof. On account of Theorem 4.2, we suppose the contrary that $N_{5}$ can be embedded into $A X$ as its sublattice, i.e. there exist elements $x, y, z \in X$ such that $x$ and $y$ are comparable, say $x>y$, and the pairs $x, z$ and $y, z$ are incomparable. In addition, the 
following equalities hold: $x \cap z=y \cap z$ and $x \cup z=y \cup z$. It can be seen that there exists $a \in G$ such that all elements $x, y, z, x \cap z, x \cup z \in L H(a)$.

Suppose that $x=h_{n} \ldots h_{1} a, y=k_{m} \ldots k_{1} a, z=l_{p} \ldots l_{1} a$ are canonical representations of $x, y, z$ w.r.t. $a$, respectively. By Theorem 3.3, there exists an index $j \leq$ $\min (n, m, p)+1$ such that $h_{j^{\prime}}=k_{j^{\prime}}=l_{j^{\prime}}$ for any $j^{\prime}<j$, and at least one of the two operations $h_{j}$ and $k_{j}$ is different from $l_{j}$, say $h_{j} \neq l_{j}$. Since $x$ and $z$ are incomparable, by Theorem $3.3, h_{j}$ and $l_{j}$ must belong to the same $L H_{c}^{i}$, for some $i \in S I^{c}$. Set $w=h_{j-1} \ldots h_{1} a, \delta_{x}=h_{n} \ldots h_{j+1}, \delta_{y}=k_{m} \ldots k_{j+1}, \delta_{z}=l_{p} \ldots l_{j+1}$. If $k_{j}=l_{j}$ then $l_{j} w<h_{j} w$, by Theorem 3.3. It follows from Theorem 4.1 that $x \cup z \in L H\left(h_{j} w\right)$ and $y \cup z \in L H\left(l_{j} w\right)$, which contradicts the fact that $x \cup z=y \cup z$. Thus, $k_{j} \neq l_{j}$. If $k_{j} \notin L H_{i}^{c}$ then $k_{j} w<h_{j} w$ and, by Remark 3.1, we also have $k_{j} w<h_{j} w$, and hence, by Theorem 3.3, we obtain $y<z$, a contradiction. Thus, $k_{j} \in L H_{i}^{c}$. According to Theorem 4.1, it follows that

$$
\begin{aligned}
& \begin{array}{l}
x \cup z \in L H\left(\left(h_{j} \vee l_{j}\right) w\right), y \cup z \in L H\left(\left(k_{j} \vee l_{j}\right) w\right) \\
x \cap z \in L H\left(\left(h_{j} \wedge l_{j}\right) w\right), y \cap z \in L H\left(\left(k_{j} \wedge l_{j}\right) w\right)
\end{array} \text { if } h_{j} w>w . \\
& \begin{array}{l}
x \cup z \in L H\left(\left(h_{j} \wedge l_{j}\right) w\right), y \cup z \in L H\left(\left(k_{j} \wedge l_{j}\right) w\right) \\
x \cap z \in L H\left(\left(h_{j} \vee l_{j}\right) w\right), y \cap z \in L H\left(\left(k_{j} \vee l_{j}\right) w\right)
\end{array} \text { if } h_{j} w<w .
\end{aligned}
$$

By virtue of axiom (i)(R3), it can easily be seen that $\left(h_{j} \vee l_{j}\right) w=\left(k_{j} \vee l_{j}\right) w$ and $\left(h_{j} \wedge l_{j}\right) w=\left(k_{j} \wedge l_{j}\right) w$, since $x \cup z=y \cup z$ and $x \cap z=y \cap z$. Consequently, it follows from (ii) of Corollary 4.1 that $h_{j}=k_{j}$.

Now, we shall show that the assumption concerning the sublattice $N_{5}$ will lead to a contradiction by induction on the length $\left|\delta_{x}\right|$ of the string $\delta_{x}$ mentioned above.

We shall only prove the case $h_{j} w>w$, since the argument for the other case is similar. Assume that $\left|\delta_{x}\right|=0$. Then, it follows from Theorem 4.1 that

$$
x \cup z=\left(h_{j} \vee l_{j}\right) w \cup \delta_{z}\left(h_{j} \vee l_{j}\right) w, y \cup z=\delta_{y}\left(h_{j} \vee l_{j}\right) w \cup \delta_{z}\left(h_{j} \vee l_{j}\right) w
$$

and

$$
x \cap z=\left(h_{j} \wedge l_{j}\right) w \cap \delta_{z}\left(h_{j} \wedge l_{j}\right) w, y \cap z=\delta_{y}\left(h_{j} \wedge l_{j}\right) w \cap \delta_{z}\left(h_{j} \wedge l_{j}\right) w .
$$

Suppose that $\left(h_{j} \vee l_{j}\right) w$ is a fixed point. By Proposition 3.2, hw is a fixed point, for every $h \in L H_{i}^{c}$. By virtue of Theorem 3.3, it follows that $\{x, y, z, x \cup z, y \cap x\}$ is isomorphic to $\left\{h_{j} w, k_{j} w, l_{j} w,\left(h_{j} \vee l_{j}\right) w,\left(k_{j} \vee l_{j}\right) w\right\}$, which contradicts the fact that $L H[w]$ is distributive by (ii) of Corollary 4.1. Now suppose that $\left(h_{j} \vee l_{j}\right) w,\left(h_{j} \wedge l_{j}\right) w$ are not fixed points. So, if $\left(h_{j} \vee l_{j}\right) w=\delta_{z}\left(h_{j} \vee l_{j}\right) w$ then $\left|\delta_{z}\right|=0$, and hence, $x \cup z=\left(h_{j} \vee l_{j}\right) w$, $x \cap z=\left(h_{j} \wedge l_{j}\right) w$. Since $x>y$ and $h_{j}=k_{j}$, it follows from (iii), Proposition 3.4 that $\left(h_{j} \vee l_{j}\right) w>\delta_{y}\left(h_{j} \vee l_{j}\right) w$ and $\left(h_{j} \wedge l_{j}\right) w>\delta_{y}\left(h_{j} \wedge l_{j}\right) w$. Thus, by Theorem 4.1, $y \cap z=\delta_{y}\left(h_{j} \wedge l_{j}\right) w$ and, hence, $x \cap z=\left(h_{j} \wedge l_{j}\right) w>y \cap z$, contrary to assumption.

If $\left(h_{j} \vee l_{j}\right) w>\delta_{z}\left(h_{j} \vee l_{j}\right) w$ then, by (iii) Proposition 3.4, $\left(h_{j} \wedge l_{j}\right) w>\delta_{y}\left(h_{j} \wedge l_{j}\right) w$, and again by Theorem 4.1, $x \cup z=\left(h_{j} \vee l_{j}\right) w$ and $x \cap z=\delta_{z}\left(h_{j} \wedge l_{j}\right) w$. On the other hand, since $y \cap z=\delta_{y}\left(h_{j} \wedge l_{j}\right) w \cap \delta_{z}\left(h_{j} \wedge l_{j}\right) w=x \cap z$, it follows that $\delta_{y}\left(h_{j} \wedge l_{j}\right) w \geq$ $\delta_{z}\left(h_{j} \wedge l_{j}\right) w$. Also, by Proposition 3.4, it implies that $\delta_{y}\left(h_{j} \vee l_{j}\right) w \geq \delta_{z}\left(h_{j} \vee l_{j}\right) w$, which yields $y \cup z=\delta_{y}\left(h_{j} \vee l_{j}\right) w<\left(h_{j} \vee l_{j}\right) w=x \cup z$, contrary to assumption.

By an analogous argument, the assumption $\left(h_{j} \vee l_{j}\right) w<\delta_{z}\left(h_{j} \vee l_{j}\right) w$ also leads to a contradiction. This concludes the proof of the case where $\left|\delta_{x}\right|=0$. 
Now suppose that a contradiction will follow for all elements $x, y$ and $z$ satisfying the mentioned assumption and, as well, the condition $\left|\delta_{x}\right|<i$. Let us consider $x, y$ and $z$, which satisfy this assumption as well as the equality $\left|\delta_{x}\right|=i$. It follows from Theorem 4.1 that

and

$$
x \cup z=\delta_{x}\left(h_{j} \vee l_{j}\right) w \cup \delta_{z}\left(h_{j} \vee l_{j}\right) w, y \cup z=\delta_{y}\left(h_{j} \vee l_{j}\right) w \cup \delta_{z}\left(h_{j} \vee l_{j}\right) w
$$$$
x \cap z=\delta_{x}\left(h_{j} \wedge l_{j}\right) w \cap \delta_{z}\left(h_{j} \wedge l_{j}\right) w, y \cap z=\delta_{y}\left(h_{j} \wedge l_{j}\right) w \cap \delta_{z}\left(h_{j} \wedge l_{j}\right) w
$$

since $h_{j} w>w$.

Let $x^{\prime}=\delta_{x}\left(h_{j} \vee l_{j}\right) w, y^{\prime}=\delta_{y}\left(h_{j} \vee l_{j}\right) w$ and $z^{\prime}=\delta_{z}\left(h_{j} \vee l_{j}\right) w$. By the assumption made on $x, y$ and $z$, and by Proposition 4.1, it can be seen that $x^{\prime}, y^{\prime}$ and $z^{\prime}$ also satisfy the assumption like that made on $x, y$ and $z$. Then, by an analogous argument as at the beginning of the proof, it follows that there exists an index $j^{\prime}$ satisfying $j<j^{\prime} \leq$ $\min (n, m, p)+1$ such that $h_{j^{\prime \prime}}=k_{j^{\prime \prime}}=l_{j^{\prime \prime}}$ for any $j^{\prime \prime}<j^{\prime}$, and $k_{j^{\prime}}=h_{j^{\prime}} \neq l_{j^{\prime}}$ and, moreover, $h_{j^{\prime}}, l_{j^{\prime}} \in L H_{i^{\prime}}^{c}$, for some $i^{\prime} \in S I^{c}$.

Set $w^{\prime}=h_{j^{\prime}-1} \ldots h_{j+1}\left(h_{j} \vee l_{j}\right) w, \delta_{x}^{\prime}=h_{n} \ldots h_{j^{\prime}+1}, \delta_{y}^{\prime}=k_{m} \ldots k_{j^{\prime}+1}, \delta_{z}^{\prime}=l_{p} \ldots l_{j^{\prime}+1}$.

Note that $\left|\delta_{x}^{\prime}\right|<i$, and, hence, according to the induction hypothesis, it leads to a contradiction. This shows that $N_{5}$ cannot be embedded into $A X$ as its sublattice.

Similarly, we can prove that $M_{5}$ cannot be embedded into $A X$ as its sublattice, as well. This concludes the proof.

5. Symmetrical RHA. In this section we prepare an algebraic foundation to investigate fuzzy logic, based on an algebraic point of view. As we know, L.A. Zadeh introduced and examined fuzzy logic based on the notion of linguistic variables. A linguistic variable of Truth is characterised by a quintuple (Truth, $T$ (Truth), $U, G, M$ ), where Truth is the name of the variable; $T$ (Truth) denotes the term-set of Truth, $U$ is a universe of discourse of the base variable, i.e. the unit interval $[0,1], G$ is a syntactic rule for generating linguistic terms of $\mathrm{T}$ (Truth), and $M$ is a semantic rule which is a mapping assigning to each linguistic term a fuzzy set on $U$. In our approach, each term is associated with an element in an RHA, and its meaning is expressed through the structure of such an RHA. Intuitively, we can recognise some what of symmetricity of the set T(Truth) and therefore, we have to examine the so-called symmetrical RHAs.

In natural languages there are many linguistic variables, which have only two distinct primary terms. These terms have intuitive contradictory meaning such as 'true' and 'false', 'old' and 'young', 'large' and 'small', 'tall' and 'short', etc. This suggested Ho and Wechler to investigate in [14] extended hedge algebras (EHAs) with exactly two generators, one of which is called positive generator, denoted by $t$, and the other is called negative generator, denoted by $f$. The positive and negative generators are characterised by $V t \geq t, V f \leq f$ and $t>f$. Under such a normalisation, it seems reasonable to consider 'true', 'old', 'large' and 'tall' as positive generators and 'false', 'young', 'small' and 'short' as negative ones.

In this section we shall also examine RHA with exactly one positive and one negative generator. Let an RHA $A X=(X, G, L H, \leq)$ be given, where the set $G$ of generators consists of one positive and one negative generator, $G=\{t, f\}$. For every $x$ in $X$, we define a so-called contradictory element of the element $x$ as follows: 
Assume that $x=h_{n} \ldots h_{1} c$, where $c \in G$, is a representation of $x$ with respect to c. An element $y$ is said to be a contradictory element of $x$ if it can be represented as $h_{n} \ldots h_{1} c^{\prime}$, with $c^{\prime} \in G$ and $c^{\prime} \neq c$. For example, $y=$ 'very very false' is a contradictory element of $x=$ 'very very true'; $v=$ 'very little bad' is a contradictory element of $u=$ 'very little good'. It is obvious that a positive generator is a contradictory element of its negative one and vice-versa. By definition, it is also obvious that if $y$ is a contradictory element of $x$ then $x$ is a contradictory element of $y$.

Definition 5.1. An RHA $A X=(X, G, L H, \leq)$, where $G$ consists exactly of one positive and one negative generator, is said to be a symmetrical $R H A$ provided every element $x$ in $X$ has a unique contradictory element in $X$, denoted by $x^{-}$.

We now give a characterisation of symmetrical RHAs.

ThEOREM 5.1. A RHA $A X=(X, G, L H, \leq)$ is symmetrical iff AX satisfies the following assumption:

(SYM) For every element $x \in X, x$ is a fixed point iff $x^{-}$is a fixed point.

Proof. To prove the necessity, assume the contrary that $x$ is a fixed point and $x^{-} \neq h x^{-}$, for some $h \in L H$. By definition, $\left(x^{-}\right)^{-}=x$ and the contradictory element of $u=h x^{-}$is the element $u^{-}=h x=x$. This shows that $u$ and $x^{-}$are two distinct contradictory elements of $x$, a contradiction to the definition of symmetrical RHAs.

Now we prove the sufficiency. Assume that $A X$ satisfies the assumption (SYM). Consider an arbitrary element $x \in X$ and let $u$ and $v$ be two contradictory elements of $x$.

Suppose that $u$ and $v$ are defined by $u=h_{n} \ldots h_{1} c^{-}$and $v=k_{m} \ldots k_{1} c^{-}$, which correspond to two representations $h_{n} \ldots h_{1} c$ and $k_{m} \ldots k_{1} c$ of $x$, where $c, c^{-} \in G$ and $c \neq c^{-}$. It is known that there exists an index $i \leq \min (n, m)$ such that $h_{i} \ldots h_{1} c$ is the canonical representation of $x$ w.r.t. $c$. This implies that $h_{j}=k_{j}$ for all $j \leq i$. It is clear that if $m=n=i$ then $u=v$. If either $i<n$ or $i<m$ then $x$ is a fixed point. By the assumption (SYM), $h_{i} \ldots h_{1} c^{-}$is also a fixed point and, hence, again $u=v$, which concludes the proof.

Notice that, by Theorem 4.3 , every symmetrical RHA $A X=(X, G, L H, \leq)$ is a distributive lattice. Moreover, we have the following:

THEOREM 5.2. For every symmetrical RHA $A X=(X, G, L H, \leq)$, the following statements hold:

(i) $(h x)^{-}=h x^{-}$, for every $h \in L H$ and $x \in X$

(ii) $\left(x^{-}\right)^{-}=x$, for every $x \in X$.

(iii) $h x>x$ iff $h x^{-}<x^{-}$, for every $h \in L H$ and $x \in X$.

(iv) $h x>k x$ iff $h x^{-}<k x^{-}$, for any $h, k \in L H$ and $x \in X$.

(v) $x<y$ iff $x^{-}>y^{-}$, for any $x, y \in X$.

(vi) $(x \cup y)^{-}=x^{-} \cap y^{-}$and $(x \cap y)^{-}=x^{-} \cup y^{-}$, for any $x, y \in X$, where $\cup$ and $\cap$ stand for join and meet, respectively, in $A X$.

Proof. The assertion (i) is a direct consequence of the definition of the contradictory elements in $A X$. Assertion (ii) follows immediately from the fact that, for every $x \in X$, $x^{-}$is uniquely defined and $x$ is a contradictory element of $x^{-}$. 
Now we shall prove assertion (iii) by induction on the length of the canonical representations of $x$ w.r.t. a generator:

Let $|x|=1$, where $|x|$ denotes the length of the canonical representation of $x$ w.r.t. $a$ generator $c$. Clearly, $x=c \in G$. If $V c>c$ and $h c>c$ then $V$ and $h$ are compatible. Thus, the inequality $h c^{-}<c^{-}$follows from the fact that $V c^{-}<c^{-}$. If $V c<c$ and $h c>c$ then $V$ and $h$ are converse. Hence, $V c^{-}>c^{-}$implies $h c^{-}<c^{-}$. For the other cases, the proof is similar. Therefore, the assertion (iii) is true for $|x|=1$. Assume that (iii) holds for all $x$ satisfying $|x|<i$. Let $u=h x$ with $|u|=i$ and consider the case that $k h x>h x$. If $k$ is positive w.r.t. $h$, then $h x>x$ and, by the induction hypothesis, $h x^{-}<x^{-}$. Hence, it implies that $k h x^{-}<h x^{-}$, since the equality cannot occur, by assumption (SYM). By the same argument, it can be proved that $k u^{-}<u^{-}$implies $k u>u$.

Analogously, we can prove (iii) for the other cases.

Now, we prove (iv). If $h$ and $k$ are converse, then $h x>x>k x$ and by (iii) it implies that $h x^{-}<x^{-}<k x^{-}$. If $h$ and $k$ are compatible then $h x>k x>x$, which implies $h>k$ in $L H^{c}+I$. Since, by (iii), we have $k x^{-}<x^{-}$and, hence, $h x^{-}<k x^{-}$.

Note that, as above, the equality $h x^{-}=k x^{-}$does not occur, since in the contrary case, $x^{-}$is a fixed point and, hence, so is its contradictory element $x$, by (SYM).

The proof for the two last assertions will be more complicated. First, we prove (v). It is known that if $x \in L H(c)$ and $y \in L H\left(c^{\prime}\right)$, with $c \neq c^{\prime}$, then $c>c^{\prime}$ can follow from $x>y$. By definition, $x^{-} \in L H\left(c^{\prime}\right)$ and $y^{-} \in L H(c)$ and, hence, $x^{-}<y^{-}$.

Suppose that $x, y \in L H(c)$ and $x>y$, and $x=h_{n} \ldots h_{1} w, y=k_{m} \ldots k_{1} w$ are, respectively, the canonical representation of $x$ and $y$ w.r.t. $w$, where $w \in L H(c)$ and $h_{1} \neq k_{1}$. Note that one of $h_{1}$ and $k_{1}$ may be the identity $I$. From $x>y$ it follows that $h_{1} w>k_{1} w$, by Theorem 3.3 and, by (iv), we have $h_{1} w^{-}<k_{1} w^{-}$.

Without loss of generality, we assume $h_{1} \neq I$ and shall prove the necessity of (v) by induction on the length of the string $\sigma=h_{n} \ldots h_{1}$, denoted by $|\sigma|$.

First consider the case when $|\sigma|=1$, i.e. $x=h_{1} w>y=k_{m} \ldots k_{1} w$. If there is no index $i$ in $S I^{c}$ such that both $h_{1}$ and $k_{1}$ belong to $L H_{i}^{c}$, then from the fact $h_{1} w^{-}<k_{1} w^{-}$ above it follows that $x=h_{1} w^{-}<y^{-}$, by (1), Theorem 3.3. In the opposite case, i.e. there exists an index $i \in S I^{c}$ such that $h_{1}, k_{1} \in L H_{i}^{c}$ and also by (1), Theorem 3.3, from the fact $x>y$ it follows that $h_{1} w \geq k_{m} \ldots k_{2} h_{1} w$. If $x=h_{1} w=k_{m} \ldots k_{2} h_{1} w$ occurs in the last inequality, then $x$ is a fixed point and, hence, $k_{1} w$ is also a fixed point, i.e. $y=k_{1} w$. Then, by (SYM), $h_{1} w^{-}$and $k_{1} w^{-}$are also fixed points. Thus, $x^{-}=h_{1} w^{-}<k_{1} w^{-}=y^{-}$. If $h_{1} w>k_{m} \ldots k_{2} h_{1} w$, then $k_{2} \neq I$ and, by Theorem 3.3, we have $h_{1} w>k_{2} h_{1} w$. Hence, it follows from (iii) that $h_{1} w^{-}<k_{2} h_{1} w^{-}$. Again by Theorem 3.3, the last inequality implies $h_{1} w^{-}<k_{m} \ldots k_{2} h_{1} w^{-}$. Moreover, by (R3), we have $k_{m} \ldots k_{2} h_{1} w^{-}<k_{m} \ldots k_{2} k_{1} w^{-}$and so, $x^{-}=h_{1} w^{-}<k_{m} \ldots k_{2} h_{1} w^{-}<k_{m} \ldots k_{2} k_{1} w^{-}=y^{-}$, which is what we require to prove for the case $|\sigma|=1$.

Now let us assume the induction hypothesis, that $x=h_{n} \ldots h_{1} w>y=k_{m} \ldots k_{1} w$ implies that $x^{-}=h_{n} \ldots h_{1} w^{-}>y^{-}=k_{m} \ldots k_{1} w^{-}$for all strings of hedges $\sigma$ satisfying $|\sigma|<p$, and for any $w \in L H(c)$. To prove the induction conclusion let us consider $x=h_{p} \ldots h_{1} w$, i.e. $|\sigma|=p$.

If there is no index $i$ in $S I^{c}$ such that both $h_{1}$ and $k_{1}$ belong to $L H_{i}^{c}$, then from $h_{1} w^{-}<k_{1} w^{-}$it follows that $x^{-}<y^{-}$, by (1), Theorem 3.3. If there exists an index 
$i$ in $S I^{c}$ such that both $h_{1}$ and $k_{1}$ belong to $L H_{i}^{c}$, then from $x=h_{p} \ldots h_{1} w>y=$ $k_{m} \ldots k_{1} w$, it follows, by Theorem 3.3 , that $x=h_{p} \ldots h_{1} w \geq k_{m} \ldots k_{2} h_{1} w$. Putting $y_{1}=k_{m} \ldots k_{2} h_{1} w$, it is clear that $y_{1}>y$, by (R3). Since $h_{1} w^{-}<k_{1} w^{-}$, as a consequence of (R3), we have $y_{1}^{-}<y^{-}$. Applying Theorem 3.3 to two elements $x$ and $y_{1}$, it follows that there exists an index $j$ such that $2 \leq j \leq \min (p, m)+1$ and $h_{j^{\prime}}=k_{j^{\prime}}$, for $2 \leq j^{\prime}<j$. If $x=y_{1}$ then we have $p=m=j$ and $h_{j} w_{j}=k_{j} w_{j}$, where $w_{j}=h_{j-1} \ldots h_{1} w$. Therefore, we have $h_{j} w_{j}^{-}=k_{j} w_{j}^{-}$, by (iv). Hence, $x^{-}=h_{j} w_{j}^{-}=$ $k_{j} w_{j}^{-}=y_{1}^{-}<y^{-}$.

Assume that $x>y_{1}$, by Theorem 3.3, it follows that $h_{j} w_{j}>k_{j} w_{j}$, and, thus, $h_{j} w_{j}^{-}<$ $k_{j} w_{j}^{-}$, by (iv). Note that the length of the string $\sigma^{\prime}=h_{p} \ldots h_{j+1}$ is less than or equal to $p$. Therefore, by the induction hypothesis, it follows that $x^{-}=h_{p} \ldots h_{j+1} h_{j} w_{j}^{-}<y_{1}^{-}=$ $k_{n} \ldots k_{j+1} k_{j} w_{j}^{-}$. Consequently, we have $x^{-}<y^{-}$. On account of (ii), it is evident that the sufficiency of $(\mathrm{v})$ can be deduced directly from the necessity. This concludes the proof of $(\mathrm{v})$.

To prove (vi), we find first, by (v), that $x=y$ iff $x^{-}=y^{-}$and that $x$ and $y$ are incomparable iff $x^{-}$and $y^{-}$are incomparable. We shall prove the validity of $(x \cup y)^{-}=$ $x^{-} \cap y^{-}$. The proof for $(x \cap y)^{-}=x^{-} \cup y^{-}$can be obtained by duality.

If $x$ and $y$ are comparable then the assertion follows directly from (v). Suppose that $x$ and $y$ are incomparable and $x=h_{n} \ldots h_{1} w, y=k_{m} \ldots k_{1} w$ are, respectively, the canonical representation of $x$ and $y$ w.r.t. $w$, where $w \in L H(c)$, for some $c \in G$, such that $h_{1} \neq k_{1}$. We shall prove the assertion by induction on the length of the string $\sigma=h_{n} \ldots h_{1}$, denoted by $|\sigma|$.

First, let us suppose that $|\sigma|=1$, i.e. $x=h_{1} w$. By Theorem 3.3, it follows that there exists an index $i$ in $S I^{c}$ such that both $h_{1}$ and $k_{1}$ belong to $L H_{i}^{c}$. By Theorem 4.1, we have

$$
x \cup y=\left\{\begin{array}{l}
\left(h_{1} \vee k_{1}\right) w \cup k_{m} \ldots k_{2}\left(h_{1} \vee k_{1}\right) w \text { if } h_{1} w>w, \\
\left(h_{1} \wedge k_{1}\right) w \cup k_{m} \ldots k_{2}\left(h_{1} \wedge k_{1}\right) w \text { if } h_{1} w<w .
\end{array}\right.
$$

Recall that $L H_{i}^{c}$ is a sublattice of $L H^{c}+I$. Hence, if $h_{1} w>w$ then $\left(h_{1} \vee k_{1}\right) w>w$. If $h_{1} w$ is a fixed point then so are $\left(h_{1} \vee k_{1}\right) w$ and $k_{1} w$, by Proposition 3.1. Hence, $y=k_{1} w$ and $x \cup y=\left(h_{1} \vee k_{1}\right) w$. Furthermore, by (SYM), it follows that $h_{1} w^{-}, k_{1} w^{-}$, $\left(h_{1} \vee k_{1}\right) w^{-}$are also fixed points, i.e. $x^{-}=h_{1} w^{-}, y^{-}=k_{1} w^{-}$. On the other hand, by (iii), it follows from $h_{1} w>w$ that $h_{1} w^{-}<w^{-}$. Thus, by Theorem 4.1, we have $x^{-} \cap y^{-}=\left(h_{1} \vee k_{1}\right) w^{-}=(x \cup y)^{-}$

Now assume that $h_{1} w$ is not a fixed point. If $k_{2}$ is positive w.r.t. $\left(h_{1} \vee k_{1}\right)$, then $k_{2}\left(h_{1} \vee\right.$ $\left.k_{1}\right) w>\left(h_{1} \vee k_{1}\right) w$. Notice that equality cannot occur, since if $k_{2}\left(h_{1} \vee k_{1}\right) w=\left(h_{1} \vee k_{1}\right) w$, then $\left(h_{1} \vee k_{1}\right) w$ is a fixed point and, hence, so is $h_{1} w$, a contradiction. By Theorem 3.3, we have $k_{m} \ldots k_{2}\left(h_{1} \vee k_{1}\right) w>\left(h_{1} \vee k_{1}\right) w$, which yields $x \cup y=k_{m} \ldots k_{2}\left(h_{1} \vee k_{1}\right) w$. By definition, we have $(x \cup y)^{-}=k_{m} \ldots k_{2}\left(h_{1} \vee k_{1}\right) w^{-}$. On the other hand, by (iii), from $k_{2}\left(h_{1} \vee k_{1}\right) w>\left(h_{1} \vee k_{1}\right) w$ it follows that $k_{2}\left(h_{1} \vee k_{1}\right) w^{-}<\left(h_{1} \vee k_{1}\right) w^{-}$. Consequently, $k_{m} \ldots k_{2}\left(h_{1} \vee k_{1}\right) w^{-}<\left(h_{1} \vee k_{1}\right) w^{-}$, by Theorem 3.3. Thus, $x^{-} \cap y^{-}=k_{m} \ldots k_{2}\left(h_{1} \vee k_{1}\right) w^{-}$, which is the requirement.

Since the proof for the case $h_{1} w<w$ is similar, this concludes the proof for the case $|\sigma|=1$ 
Now, let us suppose that $(x \cup y)^{-}=x^{-} \cap y^{-}$holds for all $x$ and $y$ with the string $\sigma$ of hedges satisfying $|\sigma|<p$, and with any $w \in L H(c)$. We shall prove the induction conclusion for $x=h_{p} \ldots h_{1} w$, where $|\sigma|=p$.

Since $x$ and $y$ are incomparable, it follows from Theorem 3.3 that there exists an index $i$ in $S I^{c}$ such that both $h_{1}, k_{1} \in L H_{i}^{c}$. Moreover, by Theorem 4.1, we have

$$
x \cup y=\left\{\begin{array}{l}
h_{p} \ldots h_{2}\left(h_{1} \vee k_{1}\right) w \cup k_{m} \ldots k_{2}\left(h_{1} \vee k_{1}\right) w \text { if } h_{1} w>w, \\
h_{p} \ldots h_{2}\left(h_{1} \wedge k_{1}\right) w \cup k_{m} \ldots k_{2}\left(h_{1} \wedge k_{1}\right) w \text { if } h_{1} w<w .
\end{array}\right.
$$

First, assume that $h_{1} w>w$. By (iii), $h_{1} w^{-}<w^{-}$and by (v), $x^{-}$and $y^{-}$are incomparable. So, on account of Theorem 4.1, we have $x^{-} \cap y^{-}=h_{p} \ldots h_{2}\left(h_{1} \vee k_{1}\right) w^{-} \cap$ $k_{m} \ldots k_{2}\left(h_{1} \vee k_{1}\right) w^{-}$, where $x^{-}=h_{p} \ldots h_{1} w^{-}$and $y^{-}=k_{m} \ldots k_{1} w^{-}$. Further, if $h_{p} \ldots h_{2}\left(h_{1} \vee k_{1}\right) w$ and $k_{m} \ldots k_{2}\left(h_{1} \vee k_{1}\right) w$ are comparable, then, by $(\mathrm{v})$, it is obvious that

$$
\begin{aligned}
(x \cup y)^{-} & =\left(h_{p} \ldots h_{2}\left(h_{1} \vee k_{1}\right) w \cup k_{m} \ldots k_{2}\left(h_{1} \vee k_{1}\right) w\right)^{-} \\
& =h_{p} \ldots h_{2}\left(h_{1} \vee k_{1}\right) w^{-} \cap k_{m} \ldots k_{2}\left(h_{1} \vee k_{1}\right) w^{-} \\
& =x^{-} \cap y^{-} .
\end{aligned}
$$

If $x_{1}=h_{p} \ldots h_{2}\left(h_{1} \vee k_{1}\right) w$ and $y_{1}=k_{m} \ldots k_{2}\left(h_{1} \vee k_{1}\right) w$ are incomparable then, by Theorem 3.3, there exists an index $j$ satisfying $2 \leq j<\min (p, m)+1$ such that $h_{j^{\prime}}=k_{j^{\prime}}$ for all $j^{\prime}$ satisfying $2 \leq j^{\prime}<j$, and there exists an index $i^{\prime}$ in $S I^{c}$ such that $h_{j}, k_{j} \in L H_{i}^{c}$. Thus, it follows from Theorem 4.1 that

$$
x_{1} \cup y_{1}=\left\{\begin{array}{l}
h_{p} \ldots h_{j+1}\left(h_{j} \vee k_{j}\right) w_{j} \cup k_{m} \ldots k_{j+1}\left(h_{j} \vee k_{j}\right) w_{j} \text { if } h_{j} w_{j}>w_{j}, \\
h_{p} \ldots h_{j+1}\left(h_{j} \wedge k_{j}\right) w_{j} \cup k_{m} \ldots k_{j+1}\left(h_{j} \wedge k_{j}\right) w_{j} \text { if } h_{j} w_{j}<w_{j},
\end{array}\right.
$$

where $w_{j}=h_{j-1} \ldots h_{2}\left(h_{1} \vee k_{1}\right) w$. Clearly, $\left|\sigma^{\prime}\right|<p$, where $\sigma^{\prime}=h_{p} \ldots h_{j+1}$. As proved above, $x_{1}^{-}$and $y_{1}^{-}$must be incomparable.

If $h_{j} w_{j}>w_{j}$ then $h_{j} w_{j}^{-}<w_{j}^{-}$, by (iii). Therefore, again by Theorem 4.1,

$$
x^{-} \cap y^{-}=h_{p} \ldots h_{j+1}\left(h_{j} \vee k_{j}\right) w_{j}^{-} \cap k_{m} \ldots k_{j+1}\left(h_{j} \vee k_{j}\right) w_{j}^{-}=x_{1}^{-} \cap y_{1}^{-} .
$$

Now, combining the obtained equalities and taking into account the induction hypothesis, we obtain $(x \cup y)^{-}=\left(h_{p} \ldots h_{j+1}\left(h_{j} \vee k_{j}\right) w_{j} \cup k_{m} \ldots k_{j+1}\left(h_{j} \vee k_{j}\right) w_{j}\right)^{-}=\left(x_{1} \cup y_{1}\right)^{-}=$ $x_{1}^{-} \cap y_{1}^{-}=x^{-} \cap y^{-}$.

For the case $h_{j} w_{j}<w_{j}$, the proof is similar.

Since the proof for the case $h_{1} w<w$, can be obtained by duality, the theorem is completely proved.

6. RHA of linguistic truth variable as an algebraic foundation of linguisticvalued logic. It is known that linguistic variables, especially linguistic truth variable, which were interpreted in the framework of fuzzy set theory by Zadeh as quintuple $(X, T(X), U, G, M)$ (see, e.g., [26-28]), have an important role in investigation of fuzzy logic and approximate reasoning methods. In the same time, the symmetrical EHA of linguistic truth variable can be taken as a basic algebraic structure for linguistic-valued fuzzy logic and linguistic reasoning methods developed in [5-7]. In the previous section, we have examined symmetrical RHAs and proved their several important properties. Particularly, all those properties hold for each RHA of linguistic truth variables. However, as an algebraic structure modelling domains of linguistic truth variable, we shall discuss 
in more detail the semantics of negation and implication and show that their properties may be appropriate for a certain fuzzy logic.

Let us consider a symmetrical RHA $A T=(T, C, L H, \leq)$ of linguistic truth variable generated by two primary generators 'True' and 'False', where 'True' is the positive generator and 'False' is the negative one, i.e. $C=\{$ True, False $\}$. For simplicity, we assume that $A T$ is finite.

It is known that the RHA $A T$ under consideration is a distributive lattice. Thus, the lattice operations of join and meet can model the semantics of the logical disjunction and conjunction. Now, we show that the operator "-" can be interpreted as a negation.

Let $A T=(T, C, L H, \leq)$ be a symmetrical RHA of linguistic truth variable, where the underlying set $T$ is defined as follows:

First we define $L H_{n}[C]$, for $n \geq 0$, by the following procedure:

$$
L H_{0}[C]=C, \quad L H_{n+1}[C]=L H\left[L H_{n}[C]\right] .
$$

Notice that, by our convention, the identity $I$ will only stand in a prefix of an expression, for instance $I \ldots I h \ldots h^{\prime} x$, and it means that if $I$ occurs explicitly in an expression, then every hedge operation applying to $I$ has no effect, i.e. $h I u=I u=u$. Therefore, it is easily seen that $C \subset L H[C] \subset L H_{2}[C] \subset \ldots \subset L H_{n}[C] \subset \ldots$ In general, this chain is infinite. However, in applications, we use only a bounded number of hedges in concatenation and, hence, we require the above chain of inclusions to be finite.

Let $p$ be a fixed positive integer. For any $x \in L H_{p}[C]$ and $x \notin L H_{p-1}[C]$, we define $h x=x$, for every $h \in L H$ and, so, we have $C \subset L H[C] \subset L H_{2}[C] \subset \ldots \subset L H_{p}[C]$. Let $T=L H_{p}[C]$. Clearly, if $A T$ is finite, then there exists $p \geq 0$ such that $T=L H_{p}[C]$. It is known that this algebra $A T$ is a complete distributive lattice. In addition, based on the properties of the unit-operation $V$, it is easy to see that the elements $V^{p}$ True and $V^{p}$ False are the greatest and least elements in $A T$ and they will be denoted by 1 and by 0 , respectively.

As observed by Ho \& Wechler in [14], the negation of vague concept may often be its contradictory concept, if it exists. For example, 'good' and 'true' are vague concepts and they involve an intuitively intended meaning. Refuting this meaning, one may often think of the meaning of the concepts 'bad' and 'false', which are the contradictory concepts of 'good' and 'true', respectively, and vice-versa. This interpretation was adopted in many investigations of fuzzy reasoning (see, e.g., [25-28]). Furthermore, it may still be possible to discuss how to refute statements containing vague concepts which are not primary concepts, for example, the concept 'Very little true'. It is natural to regard the negation of 'Very little true' as to be a concept of 'false' and it may most probably be the concept 'Very little false', a contradictory concept of the concept 'Very little true'.

Therefore, analogous to the paper [14] by Ho and Wechler, we now define the negation of an element $x$ in $A T$ to be its contradictory element, i.e. $-x=x^{-}$. This operation is called negation operation. The implication operation, denoted by $\Rightarrow$, in this algebra is defined in this paper in a regular way, i.e. by means of the negation operation and the join operation, as follows:

$$
x \Rightarrow y=\neg x \cup y \text {, for any } x \text { and } y \text { of } A T .
$$


We introduce in $A T$ a new generator $W$ defined by $L H$ (True) $>W>L H$ (False) and $h W=W$, for all $h \in L H$. This element $W$ can be understood as 'Unknown'.

Note that the algebra $A T$ with the new element $W$ also preserves all properties of the symmetrical RHA, where $W=W^{-}$. Therefore, without loss of generality we assume that the set of generators of the symmetrical RHA $A T$ consists of three elements True, $W$ and False, where $W$ is defined as above and True $>W>$ False.

Let $A T=(T, C, L H, \leq)$, with $C=\{$ True, $W$, False $\}$ and underlying set $T$ defined as above, be a symmetrical RHA of the linguistic truth variable. As examined above, the operations $\cup, \cap, \neg, \Rightarrow$ can be derived in $A T$ and, so, we can write

$$
A T=(T, C, L H, \leq, \neg, \cup, \cap, \Rightarrow, 0,1) .
$$

Throughout this section we always write simply $A T$ for such an algebra.

We are now ready to discuss some elementary properties of the negation operation and the implication operation. From the definition of these operations and Theorem 5.2, it is not difficult to see that the following holds.

THEOREM 6.1. Let AT be a symmetrical RHA of the linguistic truth variable. Then

(i) $\neg(h x)=h \neg x$, for every $h \in L H$ and $x \in T$.

(ii) $\neg(\neg x)=x$, for all $x \in T$.

(iii) $\neg(x \cup y)=\neg x \cap \neg y$ and $\neg(x \cap y)=\neg x \cup \neg y$, for all $x, y \in T$.

(iv) $x \cap \neg x \leq y \cup \neg y$, for all $x, y \in T$.

(v) $x \cap \neg x \leq W \leq x \cup \neg x$, for all $x \in T$.

(vi) $\neg 1=0, \neg 0=1$ and $\neg W=W$.

(vii) $x>y$ iff $\neg x<\neg y$, for all $x, y \in T$.

It is worth to mention that the statements (ii)-(iv) of Theorem 6.1 show that the algebra $A T$ is a Kleen algebra in the sense of Skala [24] and (vi) shows that this algebra includes the 3 -valued Łukasiewicz algebra $\{0, W, 1\}$ as its subalgebra.

As a consequence of the definition of the implication operation and Theorem 6.1 , we have the following

ThEOREM 6.2. Let AT be a symmetrical RHA of the linguistic truth variable. Then

(i) $x \Rightarrow y=\neg y \Rightarrow \neg x$.

(ii) $x \Rightarrow(y \Rightarrow z)=y \Rightarrow(x \Rightarrow z)$.

(iii) $x \Rightarrow y \geq x^{\prime} \Rightarrow y^{\prime}$ if $x \leq x$ and/or $y \geq y^{\prime}$.

(iv) $x \Rightarrow y=1$ iff either $x=0$ or $y=1$.

(v) $1 \Rightarrow x=x$ and $x \Rightarrow 1=1 ; 0 \Rightarrow x=1$ and $x \Rightarrow 0=\neg x$.

(vi) $x \Rightarrow y \geq W$ iff either $x \leq W$ or $y \geq W$, and

$x \Rightarrow y \leq W$ iff $x \geq W$ and $y \leq W$.

7. Conclusions. In this paper RHA has been introduced and investigated. We have proved that RHA with a chain of the primary generators is a distributive lattice. We would like to note that the primary generators of almost linguistic variables constitute linearly ordered sets. Furthermore, in the symmetrical RHAs of linguistic truth variable we are able to define negation operation and implication operation. Note that a method 
in linguistic reasoning based on linguistic-valued fuzzy logic corresponding to the symmetrical EHAs has been established in [7]. In this direction, we hope that it is possible to develop deductive reasoning methods based on RHAs. Remember that the symmetrical RHAs have a finer structure than that of the symmetrical EHAs and their operations may model the semantics of logical connectives more appropriately.

Some researchers, who are familiar with the fuzzy sets theory, might have some criticisms on the way we have defined negation and implication as above. In the authors' opinion, in an algebraic approach to fuzzy logic, the way we define negation, which satisfies (ii) and (vii) of Theorem 6.1, may be unique. Remember that there exists only a unique complement operation in a finite linear set, that satisfies these two properties.

The important thing which justifies the reasonableness of negation and implication is their properties, which have been proved in the algebras under consideration. Theorem 6.1 and 6.2 show that the symmetrical RHAs of the linguistic truth variable are logically rich enough to examine a kind of fuzzy logic, called linguistic-valued logic, and develop linguistic reasoning methods.

For comparison of our study with fuzzy set approach to fuzzy logics, we present roughly here a general idea of fuzzy logics based on fuzzy sets theory. As we have said previously, a basic notion to construct approximate reasoning methods is the concept of linguistic variable (see [28]), which is interpreted as quintuple $(X, T(X), U, G, M)$, where $X$ is the name of the variable; $T(X)$ denotes the term-set of $X, U$ is a universe of discourse of the base variable, $G$ is a syntactic rule for generating linguistic terms of $T(X)$, and $M$ is a semantic rule which is a mapping assigning to each linguistic term a fuzzy set on $U$, i.e. a function from $U$ into the unit interval $[0,1]$. Let us denote by $F(U,[0,1])$ the set of all functions from $U$ into the unit interval [0,1]. If $\tau$ is a linguistic value in $T(X)$ then $M(\tau) \in F(U,[0,1])$, the set of all functions from $U$ into $[0,1]$, is a meaning of $\tau$, and if, for example, a connective $O R$ occurs in $\tau$, for example $\tau=$ App.True $O R$ Poss.True, then $M(O R)$ is an operation on $F(U,[0,1])$, e.g. $M(O R)=$ Max or $M(O R)$ is a t-norm operation. So, a non-computational structure $T(X)$, from the viewpoint of fuzzy set theory, is embedded in $F(U,[0,1])$, a computational structure.

The authors emphasise that in approximate reasoning methods, the semantic mapping $M$ is rather subjective and, in applications, its reasonableness is justified by experiments. However, it is clear that there is an intuitive structure of the set $T(X)$ and, then, a question arises on mathematical point of view, whether $M$ preserves this structure, or, more exactly, whether $M$ models the intuitive structure of $T(X)$ appropriately.

Based on our study, the answer is no by the following reasons.

First, we have pointed out in the paper that $T(X)$ has a rich enough algebraic structure and $M$ does not preserve even the ordering relation of $T(X)$.

Second, from the algebraic point of view we should use the mathematical structure of the image $M(T(X)) \subseteq F(U,[0,1])$ as an underlying structure to investigate and construct fuzzy reasoning methods. However, it can be seen that this structure is too weak and, hence, one has to use the functional structure of the whole set $F(U,[0,1])$ instead of $M(T(X))$ to develop fuzzy reasoning methods, irrespectively of whether the set $F(U,[0,1])$ models the structure of $M(T(X))$ suitably or not. Note that the set $M(T(X))$ is countably infinite and in applications it is in general finite only, and we can see that the 
difference between the structures of $M(T(X))$ and $F(U,[0,1])$ is too big and, so, this may be a main reason which causes certain unreasonable questions, in the authors' opinion, and large errors in application of fuzzy reasoning methods (see $[3,16,17,19]$ ). For example, the operation MAX on fuzzy sets (as functions) in $M(T(X))$, which has to be defined meaningfully only in $F(U,[0,1])$, cannot model the connective $O R$ in natural language reasonably, especially in the case $M(T(X))$ is finite.

From this point of view, our main contribution is the following.

1. We have proved that $T(X)$ has a good enough mathematical structure, denoted by $\mathbf{A}(T(X))$ and it is also a computational structure. Particularly, the structure $\mathbf{A}(T($ Truth $))$ of linguistic variable of Truth can be considered as a rich enough logical foundation for approximate reasoning.

2. Now, linguistic variable can be interpreted as a quartuple $(X, T(X), G, M)$, where $X, T(X)$ and $G$ are the same as above, but $M$ is a mapping from $T(X)$ onto $\mathbf{A}(T(X))$ which models rather well the meaning of the terms in $T(X)$.

\section{References}

[1] G. Birkhoff, Lattice Theory (Providence, Rhode Island, 1973).

[2] S. Burris \& H. P. Sankappanavar, A Course in Universal Algebras, (Springer-Verlag: New York-Heidelberg-Berlin, 1981).

[3] Z. CAO and A. KAndel, Applicability of some fuzzy implication operators, Fuzzy Sets and Systems 31(1989), 151-186.

[4] N. CAT Ho, Generalized Post algebras and their application to some infinitary manyvalued logic, Dissertationes Math. 107 (1973) 1-76.

[5] N. Cat Ho, Fuzziness in structure of linguistic truth values: A foundation for development of fuzzy reasoning, Proc. of ISMVL 87, Boston, USA (IEEE Computer Society Press, New York ),1987, 326-335.

[6] N. CAT Ho, Linguistic-valued logic and a deductive method in linguistic reasoning, Proc. of the Fifth IFSA 93, Seoul, Korea, July 4-9, 1993.

[7] N. CAT Ho, A method in linguistic reasoning on a knowledge base representing by sentences with linguistic belief degree, Fundamenta Informaticae Vol. 28(3,4) (1996), 247-259.

[8] N. Cat Ho \& H. Rasiowa, Plain semi-Post algebras and their representability, Studia Logica 48(4) (1989), 509-530.

[9] N. CAT Ho \& H. VAN NAM, A refinement structure of hedge algebras, Proc. of the NCST of Vietnam, Vol. 9(1) (1997), 15-28.

[10] N. CAt Ho \& H. VAn NAm, Lattice character of the refinement structure of hedge algebras, J. of Comp. Sci. and Cyber., Vol. 12(1) (1996), 7-20.

[11] N. CAT Ho \& H. VAN NAM, Refinement of hedge algebras based on free distributive lattices generated by hedge operations, Research Report at Workshop on Information Technology: R \& D, IOIT, 5-6 Dec. 1996, 156-182 (in Vietnamese).

[12] N. CAT Ho \& H. VAN NAM, Refinement structure of hedge algebras: An algebraic basis for a linguistic-valued fuzzy logic, Present at Inter. Conf. on Discrete Mathematics and Allied Topics, 10-13 Nov. 1997, India. 
[13] N. Cat Ho \& W. WechleR, Hedge algebras: An algebraic approach to structure of sets of linguistic truth values, Fuzzy Sets and Systems 35(1990), 281-293.

[14] N. Cat Ho \& W. Wechler, Extended hedge algebras and their application to fuzzy logic, Fuzzy Sets and Systems 52(1992), 259-281.

[15] R. Giles, Łukasiewicz logic and fuzzy set theory, Inter. J. of Man-Machine stud. 8 (1976), 313-327.

[16] J. B. Kiszka, M. E. KochańskA and S. ŚLiwińskA, The influence of some fuzzy implication operators on the accuracy of a fuzzy model-Part I, Fuzzy Sets and Systems 15(1983), 111-128.

[17] J. B. Kiszka, M. E. Kochańska and S. Śliwińska, The influence of some fuzzy implication operators on the accuracy of a fuzzy model-Part II, Fuzzy Sets and Systems 15(1983), 223-240

[18] G. LAKOFF, Hedges: A study in meaning criteria and the logic of fuzzy concepts, J. Philos. Logic 2 (1973) 458-508 (also presented at the 8th Regional Meeting of the Chicago Linguistic Society, 1972).

[19] M. Mizumoto and H.-J. Zimmermann, Comparison of fuzzy reasoning methods, Fuzzy Sets and Systems 8(1982), 253-283.

[20] H. Rasiowa, An Algebraic Approach to Non-classical Logic (North-Holland, AmsterdamNew York, 1974).

[21] H. Rasiowa \& R. Sikorski, The Mathematics of Metamathematics, second edition (Polish Scientific Publ., Warszawa, 1968).

[22] D. B. Rinks, A heuristic approach to aggregate production scheduling using linguistic variables, Proc. of Inter. Congr. on Appl. Systems Research and Cybernetics, Vol. VI (1981) 2877-2883.

[23] R. Sikorski, Boolean Algebras, third edition, (Springer-Verlag, Berlin-Heidelberg-New York, 1969).

[24] H. J. Skala, On many-valued logics, fuzzy sets, fuzzy logics and their applications, Fuzzy Sets and Systems 1 (1978) 129-149.

[25] Y. Tsukamoto, An approach to fuzzy reasoning method, in M. M. Gupta, R. K. Ragade, R. R. Yager, Eds., Advances in Fuzzy Set Theory and Applications (North-Holland, Amsterdam, 1979) 137-149.

[26] L. A. ZADeh, Fuzzy-set-theoretic interpretation of linguistic hedges, J. of Cybernetics 2 (1972) 4-34.

[27] L. A. ZADEh, A theory of approximate reasoning, in: R. R. Yager, S. Ovchinnikov, R. M. Tong and H. T. Nguyen, Eds., Fuzzy Sets and Applications: The selected papers by L. A. Zadeh (Wiley, New York, 1987) 367-411.

[28] L. A. ZADEH, The concept of linguistic variable and its application to approximate reasoning Inform. Sci. (I) 8(1975) 199-249; (II) 8(1975) 310 -357; (III) 9(1975) 43-80. 